\title{
Observation of long-range, near-side angular correlations in proton-proton collisions at the LHC
}

\section{The CMS collaboration}

ABSTRACT: Results on two-particle angular correlations for charged particles emitted in proton-proton collisions at center-of-mass energies of $0.9,2.36$, and $7 \mathrm{TeV}$ are presented, using data collected with the CMS detector over a broad range of pseudorapidity $(\eta)$ and azimuthal angle $(\phi)$. Short-range correlations in $\Delta \eta$, which are studied in minimum bias events, are characterized using a simple "independent cluster" parametrization in order to quantify their strength (cluster size) and their extent in $\eta$ (cluster decay width). Long-range azimuthal correlations are studied differentially as a function of charged particle multiplicity and particle transverse momentum using a $980 \mathrm{nb}^{-1}$ data set at $7 \mathrm{TeV}$. In high multiplicity events, a pronounced structure emerges in the two-dimensional correlation function for particle pairs with intermediate $p_{T}$ of $1-3 \mathrm{GeV} / c, 2.0<|\Delta \eta|<4.8$ and $\Delta \phi \approx 0$. This is the first observation of such a long-range, near-side feature in two-particle correlation functions in $p p$ or $p \bar{p}$ collisions.

KEYWORDS: Hadron-Hadron Scattering 


\section{Contents}

1 Introduction 1

2 Experimental setup, triggering, and event selections $\quad 2$

3 Track selection $\quad 4$

4 Calculation of the two-particle correlation function 4

5 Corrections for tracking and event selection inefficiencies 5

5.1 Correction for tracking inefficiency 5

$\begin{array}{lll}5.2 & \text { Event selection correction for minimum bias data } & 6\end{array}$

$\begin{array}{lll}5.3 & \text { Event selection correction for high multiplicity data } & 6\end{array}$

6 Short-range correlations in $0.9,2.36$, and $7 \mathrm{TeV}$ data 6

$\begin{array}{lll}7 & \text { Long-range correlations in } 7 \mathrm{TeV} \text { data } & 13\end{array}$

8 Conclusion $\quad 18$

$\begin{array}{ll}\text { The CMS collaboration } & 22\end{array}$

\section{Introduction}

This paper presents measurements of two-particle angular correlations of charged particles emitted in proton-proton $(p p)$ collisions at center of mass energies $(\sqrt{s})$ of $0.9,2.36$, and $7 \mathrm{TeV}$. This first study of short- and long-range correlations in $p p$ collisions at the LHC high energy frontier provides important information for characterizing Quantum Chromodynamics (QCD) in this energy regime, especially the mechanism of hadronization and possible collective effects due to the high particle densities reached in these collisions. Multiparticle correlations in high energy collisions have been measured previously for a broad range of collision energies and colliding systems with the goal of understanding the underlying mechanism of particle production [1-7].

Two related studies of angular correlations have been performed using two-dimensional $\Delta \eta-\Delta \phi$ correlation functions. Here $\Delta \eta$ is the difference in pseudorapidity $\eta(=-\ln (\tan (\theta / 2))$, where $\theta$ is the polar angle relative to the beam axis) between the two particles and $\Delta \phi$ is the difference in their azimuthal angle $\phi$ (in radians). In a first analysis, $p p$ data collected with a minimum bias trigger at $0.9,2.36$, and $7 \mathrm{TeV}$ were used to study short-range correlations $(|\Delta \eta|$ less than $\approx 2)$. In a second study, the long-range structure $(2.0<|\Delta \eta|<4.8)$ of twoparticle correlation functions was examined as a function of charged particle multiplicity and particle transverse momentum for a large data set at $7 \mathrm{TeV}$. 
In short-range correlations in minimum bias events, a peak with a typical width of about one unit in $\Delta \eta$ is observed. A useful way to quantify this effect is to assume that the initial interactions emit so-called "independent clusters", which subsequently decay isotropically in their own rest frame into the observed hadrons $[1-3,5-7]$. This simple independent cluster model (ICM) parametrization of the observed correlation function allows a quantitative comparison of data and models for different collision energies and collision systems. The observed correlation strength and extent in relative pseudorapidity between the particles are parametrized by a Gaussian distribution. The fitted parameters in this ansatz are the cluster multiplicity or "size" (the average number of particles into which a cluster decays) and the decay "width" (the spread of the daughter particles in pseudorapidity). This ansatz is only a phenomenological parametrization which provides no insight as to the nature of the assumed clusters nor to the mechanisms by which clusters are formed. Relating these results to the underlying QCD dynamics requires further modeling.

To investigate long-range azimuthal correlations $(2.0<|\Delta \eta|<4.8)$, a high-statistics data set of high multiplicity $p p$ events at $7 \mathrm{TeV}$ was used. In current $p p$ Monte Carlo (MC) event generators, the typical sources of such long-range correlations are momentum conservation and away-side $(\Delta \phi \approx \pi)$ jet correlations. Measurements at the Relativistic Heavy Ion Collider (RHIC) have revealed that the long-range structure of two-particle angular correlation functions is significantly modified by the presence of the hot and dense matter formed in relativistic heavy ion collisions [7]. Several novel correlation structures over large $\Delta \eta$ were observed in azimuthal correlations for intermediate particle transverse momenta, $p_{T} \approx 1-5 \mathrm{GeV} / c[8,9]$. Since the particle densities produced in the highest multiplicity $p p$ collisions at LHC energies begin to approach those in high energy collisions of relatively small nuclei such as copper [10], it is natural to search for the possible emergence of new features in the two-particle correlation function from high multiplicity $p p$ events [1118]. Therefore, the azimuthal $(\Delta \phi)$ correlation functions from the large data set at $7 \mathrm{TeV}$ have been studied differentially by binning the events in the observed charged particle multiplicity and by selecting particle pairs in bins of the transverse momentum of the particles.

The paper is organized as follows: the experimental setup, event triggering, and event selection for both analyses are described in section 2. Criteria used to select tracks are listed in section 3. The general procedure for calculating the correlation functions and the CMS-specific efficiency corrections are described in sections 4 and 5, respectively. Results for the analysis of short-range correlations in minimum bias data using the cluster parametrization are given in section 6 . The study of long-range correlations as a function of event multiplicity and particle transverse momentum is detailed in section 7 .

\section{Experimental setup, triggering, and event selections}

This analysis used three data sets collected with $p p$ interactions at $\sqrt{s}=0.9,2.36$, and $7 \mathrm{TeV}$. A detailed description of the CMS experiment can be found in ref. [19]. The detector subsystems used for the present analysis are the pixel and silicon-strip tracker (SST), covering the region $|\eta|<2.5$ and immersed in a $3.8 \mathrm{~T}$ axial magnetic field. The lead 
tungstate crystal electromagnetic calorimeter (ECAL), the brass/scintillator hadron calorimeter (HCAL), and the forward calorimeter (HF, covering the region $2.9<|\eta|<5.2$ ), were also used for online and offline event selections. The detailed MC simulation of the CMS detector response is based on GEANT4 [20].

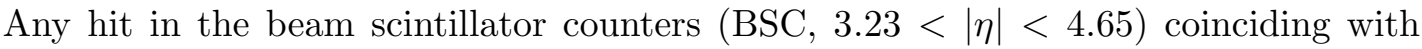
colliding proton bunches was used for triggering the data acquisition in the minimum bias trigger. To preferentially select non-single-diffractive (NSD) events, a coincidence of at least one $\mathrm{HF}$ calorimeter tower with more than $3 \mathrm{GeV}$ of total energy on each of the positive and negative sides was required. Events were also required to contain at least one reconstructed primary vertex $(\mathrm{PV})$ that fell within a $4.5 \mathrm{~cm}$ of the nominal collision point along the beam axis and within a radius of $0.15 \mathrm{~cm}$ measured perpendicular to the beam relative to the average vertex position, and to contain at least three fully reconstructed tracks associated with the primary vertex. Outside this relatively narrow vertex range, the density of events was too small to ensure enough statistics for constructing the random background distribution (section 4) in small bins of the longitudinal $(z)$ vertex position. Beam-halo and other beam-background events were rejected as described in ref. [21]. The contamination of background events after selections in the colliding-bunch data sample was found to be negligible $(<0.1 \%)$.

After all selections are applied, the total number of events used for the minimum bias analysis of cluster properties described in section 6 is $168854\left(3.3 \mu \mathrm{b}^{-1}\right)$ for $0.9 \mathrm{TeV}$, $10902\left(0.2 \mu \mathrm{b}^{-1}\right)$ for $2.36 \mathrm{TeV}$, and $150086\left(3.0 \mu \mathrm{b}^{-1}\right)$ for $7 \mathrm{TeV}$, where the numbers in parentheses are the approximate integrated luminosity for the individual data samples. The systematic uncertainties in the results shown in section 6 significantly exceed the statistical uncertainties for the $150 \mathrm{k}$ event minimum bias data sample at $7 \mathrm{TeV}$, so no further events were included in this analysis.

In order to investigate the properties of the high multiplicity $p p$ collisions, a dedicated high multiplicity trigger was designed and implemented in the two levels of the CMS trigger system. At Level 1 (L1), the total transverse energy summed over the entire set of CMS calorimeters (ECAL, HCAL, and HF) was required to be greater than $60 \mathrm{GeV}$. At the highlevel trigger (HLT), online tracks built from the three layers of pixel detectors with a track origin within a cylindrical region of $21 \mathrm{~cm}$ along the beam and $0.5 \mathrm{~cm}$ in the transverse radius were used in an online vertexing algorithm. The number of pixel tracks $\left(N_{\text {trk }}^{\text {online }}\right)$ with $|\eta|<2, p_{T}>0.4 \mathrm{GeV} / c$, and a distance of closest approach of $0.12 \mathrm{~cm}$ or less to the best vertex (the one associated with the highest number of tracks) was determined for each event. Data were taken with a threshold initially set to $N_{\text {trk }}^{\text {online }}>70$. During later, higher-luminosity running, the lower limit was raised to 85 .

The total integrated luminosity for the high multiplicity analysis was $980 \mathrm{nb}^{-1}$. The total number of events in each of the bins of offline reconstructed track multiplicity, $N_{\text {trk }}^{\text {offline }}$, used in the analysis are listed in table 1. To take advantage of the full acceptance of the

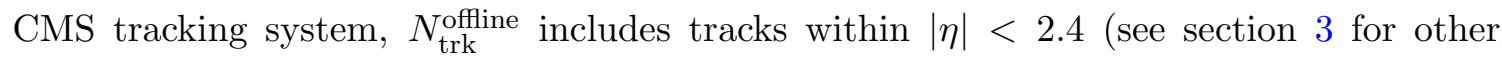
offline track selection criteria). The table also lists the average values of $N_{\text {trk }}^{\text {offline }}$ as well as the average of $N_{\text {trk }}^{\text {corrected }}$, the event multiplicity corrected for all detector and algorithm inefficiencies, as described in section 5 . 


\begin{tabular}{|l|c|r|r|}
\hline Multiplicity bin $\left(N_{\text {trk }}^{\text {offline }}\right)$ & Event Count & $\left\langle N_{\text {trk }}^{\text {offline }}\right\rangle$ & $\left\langle N_{\text {trk }}^{\text {corrected }}\right\rangle$ \\
\hline MinBias & $21.43 \mathrm{M}$ & 15.9 & 17.8 \\
$N_{\text {trk }}^{\text {offine }}<35$ & $19.36 \mathrm{M}$ & 13.0 & 14.1 \\
$35 \leq N_{\text {trk }}^{\text {offline }}<90$ & $2.02 \mathrm{M}$ & 45.3 & 53.1 \\
$90 \leq N_{\text {trk }}^{\text {offline }}<110$ & $302.5 \mathrm{k}$ & 96.6 & 111.7 \\
$N_{\text {trk }}^{\text {offline }} \geq 110$ & $354.0 \mathrm{k}$ & 117.8 & 136.1 \\
\hline
\end{tabular}

Table 1. Number of events for each multiplicity bin used in the $7 \mathrm{TeV}$ analysis with total integrated luminosity of $980 \mathrm{nb}^{-1}$. The multiplicity of offline reconstructed tracks, $N_{\text {trk }}^{\text {offline }}$, was counted within the kinematic cuts of $|\eta|<2.4$ and $p_{T}>0.4 \mathrm{GeV} / c$. The last two columns list the average values of $N_{\mathrm{trk}}^{\text {offline }}$ as well as the average of $N_{\mathrm{trk}}^{\text {corrected }}$, the event multiplicity corrected for all detector and algorithm inefficiencies.

\section{Track selection}

In this analysis, the so-called CMS highPurity [22] tracks were used. Additionally, a reconstructed track was considered as a primary-track candidate if the significance of the separation along the beam axis, $z$, between the track and the primary vertex, $d_{z} / \sigma\left(d_{z}\right)$, and the significance of the impact parameter relative to the primary vertex transverse to the beam, $d_{\mathrm{xy}} / \sigma\left(d_{\mathrm{xy}}\right)$, were each less than 3 . In order to remove tracks with potentially poorly reconstructed momentum values, the relative uncertainty of the momentum measurement, $\sigma\left(p_{T}\right) / p_{T}$, was required to be less than $10 \%$.

To ensure reasonable tracking efficiency and low fake rate, only tracks within $|\eta|<2.4$ and above a minimum $p_{T}$ value were used. For the inclusive analysis, the selected range was $0.1 \mathrm{GeV} / c<p_{T}<5.0 \mathrm{GeV} / c$. The effect of the upper limit imposed on $p_{T}$ is negligible. The effects of the lower $p_{T}$ cut, as well as the effect of the $\eta$ restriction on the determination of cluster parameters from $\Delta \eta$ correlations, are significant and will be discussed in more detail below. To avoid possible bias in the high multiplicity analysis, the lower cutoff was raised to $p_{T}>0.4 \mathrm{GeV} / c$ when classifying the event multiplicity in order to match the cut applied in the online tracking.

\section{Calculation of the two-particle correlation function}

For both minimum bias and high-multiplicity triggered collision events, the first step in extracting the correlation function was to divide the sample into bins in track multiplicity. For the minimum bias sample, 10 bins were used, each containing about the same number of events. Following an approach similar to that in refs. $[3,6]$, the $p_{T}$-inclusive charged two-particle correlation as a function of $\Delta \eta$ and $\Delta \phi$ is defined as follows:

$$
R(\Delta \eta, \Delta \phi)=\left\langle(\langle N\rangle-1)\left(\frac{S_{N}(\Delta \eta, \Delta \phi)}{B_{N}(\Delta \eta, \Delta \phi)}-1\right)\right\rangle_{\text {bins }}
$$

where $S_{N}$ and $B_{N}$ are the signal and random background distributions, defined in eqs. (4.2) and (4.3) respectively, $\Delta \eta\left(=\eta_{1}-\eta_{2}\right)$ and $\Delta \phi\left(=\phi_{1}-\phi_{2}\right)$ are the differences in pseudorapidity and azimuthal angle between the two particles, $\langle N\rangle$ is the number of tracks per 
event averaged over the multiplicity bin, and the final $R(\Delta \eta, \Delta \phi)$ is found by averaging over multiplicity bins. For simplicity in eq. (4.1) and the discussion in this section, $N$ is used to represent the total number of offline reconstructed tracks per event. Note that the order in which the particles are considered has no significance. The quantities $\Delta \eta$ and $\Delta \phi$ are always taken to be positive and used to fill one quadrant of the $\Delta \eta, \Delta \phi$ histograms with the other three quadrants filled by reflection. Therefore, the resulting distributions are symmetric about $(\Delta \eta, \Delta \phi)=(0,0)$ by construction.

For each multiplicity bin, the signal distribution:

$$
S_{N}(\Delta \eta, \Delta \phi)=\frac{1}{N(N-1)} \frac{d^{2} N^{\text {signal }}}{d \Delta \eta d \Delta \phi}
$$

was determined by counting all particle pairs within each event, using the weighting factor $N(N-1)$, then averaging over all events. This represents the charged two-particle pair density function normalized to unit integral. The background distribution:

$$
B_{N}(\Delta \eta, \Delta \phi)=\frac{1}{N^{2}} \frac{d^{2} N^{\text {mixed }}}{d \Delta \eta d \Delta \phi}
$$

denotes the distribution of uncorrelated particle pairs representing a product of two singleparticle distributions, also normalized to unit integral. This distribution was constructed by randomly selecting two different events within the same multiplicity bin and pairing every particle from one event with every particle in the other (in this case, the normalization factor $1 / N^{2}$ corresponds to $1 / N_{1} N_{2}$ event-by-event). The pairs of events used to compute the background were also required to be within the same $0.5 \mathrm{~cm}$ wide bin in the vertex location along the beam.

As indicated in eq. (4.1), the ratio of $S_{N}(\Delta \eta, \Delta \phi)$ to $B_{N}(\Delta \eta, \Delta \phi)$ was first calculated in each multiplicity bin. Dividing the background in this way corrects for detector effects such as tracking inefficiencies, non-uniform acceptance, etc. The ratio of signal to background was then weighted by the track multiplicity factor, $\langle N\rangle-1$ (where $\langle N\rangle$ is the average multiplicity in each bin), and averaged over all the multiplicity bins to arrive at the final two-particle correlation function $R(\Delta \eta, \Delta \phi)$.

\section{Corrections for tracking and event selection inefficiencies}

\subsection{Correction for tracking inefficiency}

Studies with simulated events showed that the combined geometrical acceptance and reconstruction efficiency for the global track reconstruction exceeds $50 \%$ around $p_{T} \approx 0.1 \mathrm{GeV} / c$ over the full CMS tracker acceptance $(|\eta|<2.4)$ for charged hadrons. The efficiency is greater than $90 \%$ in the $|\eta|<1$ region for $p_{T}>0.6 \mathrm{GeV} / c$. Detailed studies of tracking efficiencies using MC-based and data-based methods can be found in [23]. The tracking efficiency correction factor, $\epsilon^{\text {trk }}$, was determined by taking the ratio of the number of reconstructed tracks $\left(N^{\text {trk }}\right)$ to that of generator level primary charged particles $\left(N^{\text {gen }}\right)$ in the simulated $\mathrm{MC}$ events as a function of $p_{T}, \eta, z_{\mathrm{vtx}}$ :

$$
\epsilon^{\mathrm{trk}}\left(\eta, p_{T}, z_{\mathrm{vtx}}\right)=\frac{N^{\mathrm{trk}}\left(\eta, p_{T}, z_{\mathrm{vtx}}\right)}{N^{\operatorname{gen}}\left(\eta, p_{T}, z_{\mathrm{vtx}}\right)}
$$


In constructing the signal and background distributions, this correction was applied as an inverse weight, $1 / \epsilon^{\mathrm{trk}}\left(\eta, p_{T}, z_{\mathrm{vtx}}\right)$, to each particle. After this correction, the two-particle correlation function found using reconstructed tracks from simulated events matched that obtained at the generator level to within $1.4 \%$.

Using simulations, the tracking efficiency was found to have little or no dependence on multiplicity within the range studied in the present work. The fake rate did increase slightly with multiplicity but remained at the 1-2\% level. Therefore, the corrections applied for tracking efficiency and fake rate were independent of event multiplicity.

\subsection{Event selection correction for minimum bias data}

For the minimum bias data, inefficiencies in triggering and vertex reconstruction of low multiplicity events resulted in multiplicity distributions of reconstructed tracks which were biased toward higher average values. The correction factor for this effect, $\epsilon^{\text {evt }}$, was determined by taking the ratio of two generator-level MC multiplicity distributions, one with offline event selection applied $\left(N_{\text {gen }}^{\text {evtSel }}\right)$ and one for all NSD MC events $\left(N_{\text {gen }}^{\mathrm{NSD}}\right)$. In eq. $(5.2)$, $N_{\text {trk }}^{\text {true }}$ represents the true number of particles in the event. The NSD event selection efficiency:

$$
\epsilon^{\text {evt }}\left(N_{\text {trk }}^{\text {true }}\right)=\frac{N_{\text {gen }}^{\text {evtSel }}\left(N_{\text {tru }}^{\text {true }}\right)}{N_{\text {gen }}^{\text {NSD }}\left(N_{\text {trk }}^{\text {true }}\right)}
$$

is about $50 \%$ at $N_{\text {trk }}^{\text {true }}=6$ and reaches $100 \%$ around $N_{\text {trk }}^{\text {true }}=15$. When calculating the correlation function, each event was weighted by the inverse of the event selection efficiency evaluated at $N_{\text {trk }}^{\text {corrected }}$ which is the number of particles corrected for acceptance and tracking efficiency as described above, $1 / \epsilon^{\mathrm{evt}}\left(N_{\mathrm{trk}}^{\text {corrected }}\right)$.

\subsection{Event selection correction for high multiplicity data}

The two high-multiplicity trigger thresholds used in the HLT (see section 2) give different trigger efficiencies. Distributions for offline reconstructed track multiplicity, $N_{\text {trk }}^{\text {offline }}$, in minimum bias and high multiplicity triggered events at $7 \mathrm{TeV}$ are shown in the top panels of figure 1. Kinematic cuts of $|\eta|<2.4$ and $p_{T}>0.4 \mathrm{GeV} / c$ were used in defining $N_{\text {trk }}^{\text {offline }}$ (see section 3 for other offline track selections). The statistics of events with $N_{\text {trk }}^{\text {offline }} \geq 110$ were enhanced by a factor of about 1000 with the high multiplicity trigger relative to the minimum bias trigger due the large prescale factor applied to the latter sample. The lower panels in figure 1 show the HLT efficiency, obtained from data, of the two high multiplicity triggers relative to the minimum bias trigger. The L1 triggering efficiency (not shown in figure 1) is not a concern since it reaches $100 \%$ efficiency for events with $N_{\text {trk }}^{\text {offine }} \geq 90$. A weight given by the inverse of the HLT efficiency, $\epsilon_{\mathrm{evt}}^{H L T}\left(N_{\mathrm{trk}}^{\mathrm{offline}}\right)$, was applied to all pairs from a given event.

\section{Short-range correlations in $0.9,2.36$, and $7 \mathrm{TeV}$ data}

The final two-particle inclusive correlation functions are shown in figure 2 as a function of $\Delta \eta$ and $\Delta \phi$ at $\sqrt{s}=0.9,2.36$, and $7 \mathrm{TeV}$. A small region with $|\Delta \eta|<0.06$ and $|\Delta \phi|<$ 


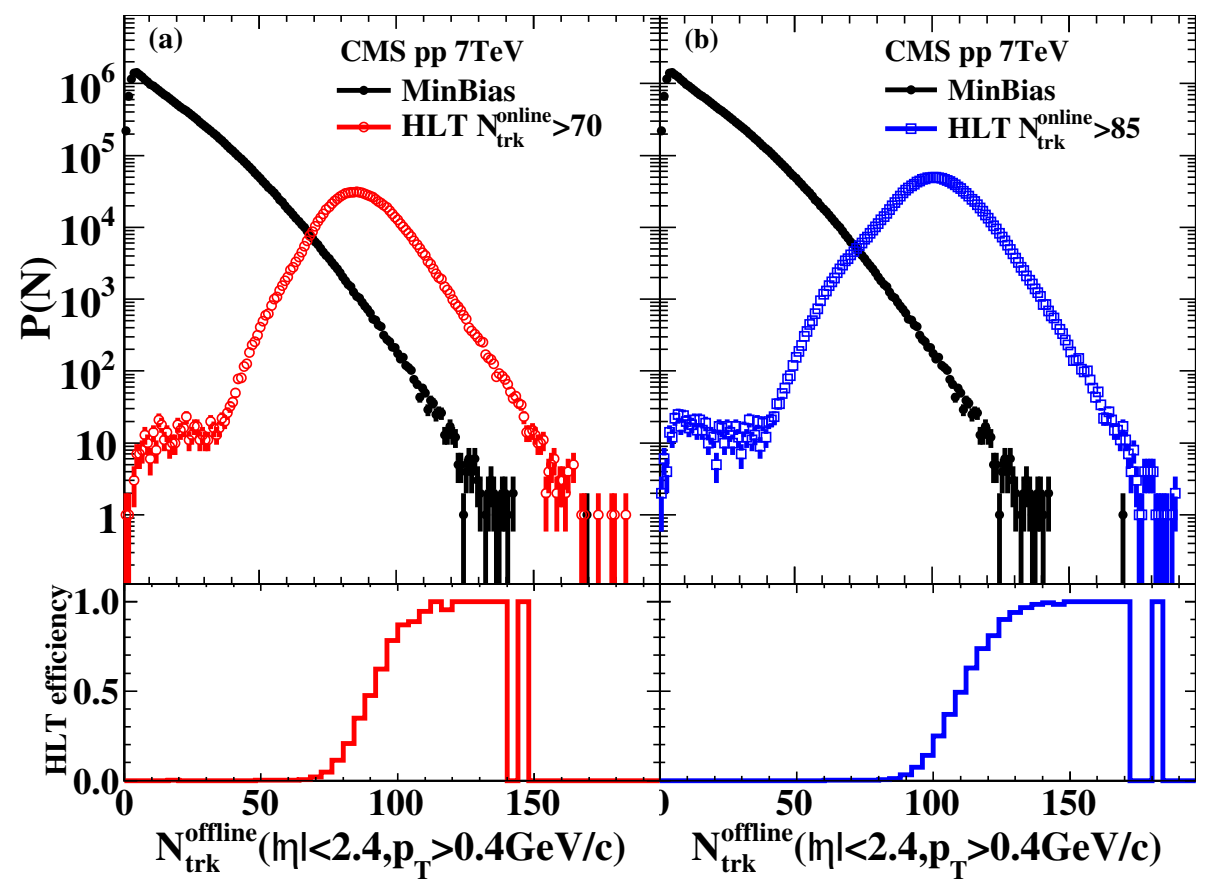

Figure 1. Distributions of the number of tracks reconstructed in offline analysis, $N_{\text {trk }}^{\text {offine }}$, for minimum bias events, as well as high-multiplicity triggered events, both at $7 \mathrm{TeV}$, with online multiplicity $N_{\text {trk }}^{\text {online }}$ greater than (a) 70 and (b) 85. The total integrated luminosity of the data set is $980 \mathrm{nb}^{-1}$. The minimum bias trigger was heavily prescaled during higher luminosity LHC running. The HLT efficiency turn-on curves for the two high multiplicity triggers are shown in the two panels at the bottom.
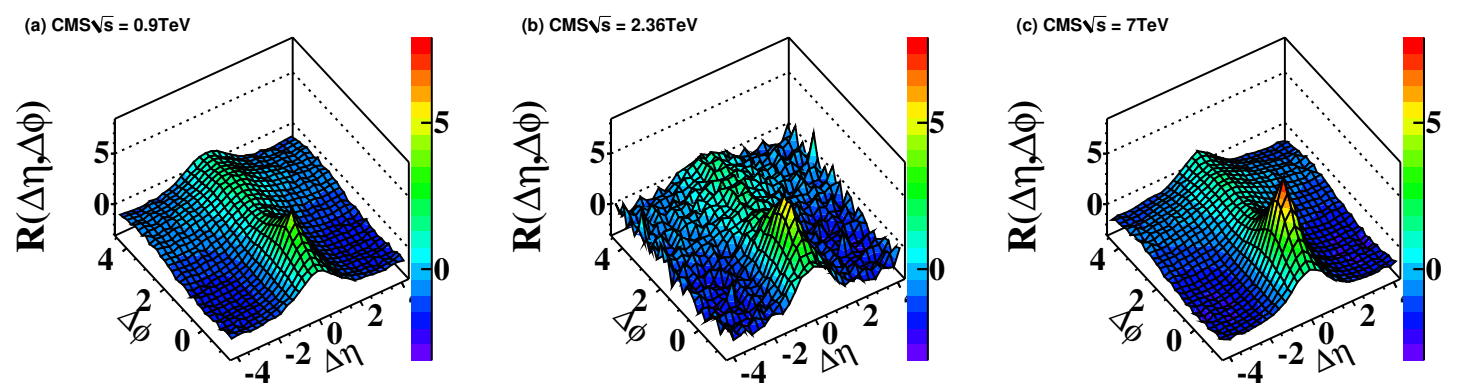

Figure 2. Two-particle correlation functions versus $\Delta \eta$ and $\Delta \phi$ in $p p$ collisions at $\sqrt{s}=($ a) 0.9 , (b) 2.36 , and (c) $7 \mathrm{TeV}$.

0.06 was excluded in both signal and background distributions in order to reject residual secondary effects (i.e., any tracks from photon conversions, weak decays, or $\delta$-electrons which were not rejected by the cut on the projected distance of the track from the vertex).

The complex two-dimensional (2-D) correlation structure shown in figure 2 is dominated by three prominent components: a narrow peak at $(\Delta \eta, \Delta \phi) \approx(0,0)$ which can be 

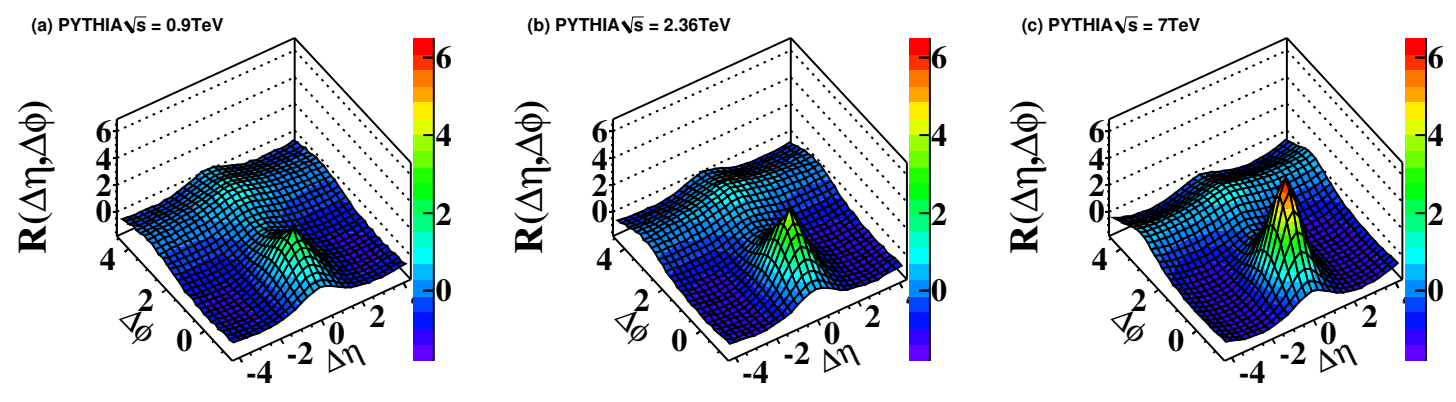

Figure 3. Two-particle correlation functions versus $\Delta \eta$ and $\Delta \phi$ in PYTHIA D6T tune at $\sqrt{s}=$ (a) 0.9 , (b) 2.36 , and (c) $7 \mathrm{TeV}$.

understood as the contribution from higher $p_{T}$ clusters (e.g., hard processes like jets); a ridge at $\Delta \phi \approx \pi$ spread over a broad range in $\Delta \eta$, interpreted as due to away-side jets or more generally momentum conservation; and an approximately Gaussian ridge at $\Delta \eta \approx 0$ extending over the whole range of $\Delta \phi$, becoming broader toward larger $\Delta \phi$ values, which arises from the decay of clusters with lower $p_{T}$ (e.g., soft QCD string fragmentation). This broadening will be discussed in quantitative detail later in this section. The PHOBOS experiment at RHIC observed similar correlation structures in $p p$ collisions at $\sqrt{s}=200 \mathrm{GeV}$ and $410 \mathrm{GeV}$ [6]. Qualitatively similar structures also exist in PYTHIA (figure 3 for D6T tune [24]) although they do not reproduce the strength of the correlations seen in the data. The qualitative features of the observed correlations in the data are also consistent with an independent cluster approach according to a simulation study from the ISR experiment using a low-mass resonance $(\rho, \omega, \eta)$ gas model [3] and a MC model of isotropic cluster decays from the PHOBOS experiment [7]. Bose-Einstein Correlations (BEC, also known as the Hanbury-Brown and Twiss effect [25]) have been measured in $p p$ collisions [26-28] but their influence on the extracted cluster parameters has been found to be negligible [6].

To quantify one aspect of the correlation structure, the 2-D correlation functions were reduced to one-dimensional (1-D) functions of $\Delta \eta$ by integrating $S_{N}(\Delta \eta, \Delta \phi)$ and $B_{N}(\Delta \eta, \Delta \phi)$ over $\Delta \phi:$

$$
R(\Delta \eta)=\left\langle(\langle N\rangle-1)\left(\frac{\int S_{N}(\Delta \eta, \Delta \phi) d \Delta \phi}{\int B_{N}(\Delta \eta, \Delta \phi) d \Delta \phi}-1\right)\right\rangle_{\mathrm{bins}}
$$

The 1-D two-particle pseudorapidity correlation functions, $R(\Delta \eta)$, where $\Delta \phi$ was averaged over the entire range from 0 to $\pi$, are shown for all three energies in figure 4 .

In the context of an ICM description, $R(\Delta \eta)$ can be parametrized using the functional form [2]:

$$
R(\Delta \eta)=\alpha\left[\frac{\Gamma(\Delta \eta)}{B(\Delta \eta)}-1\right]
$$

where the correlation strength $\alpha=\frac{\langle K(K-1)\rangle}{\langle K\rangle}$ depends on the average numbers of particles into which a cluster decays, the cluster size $K$. The function $\Gamma(\Delta \eta)$ is a Gaussian function proportional to

$$
\exp \left[-(\Delta \eta)^{2} /\left(4 \delta^{2}\right)\right]
$$



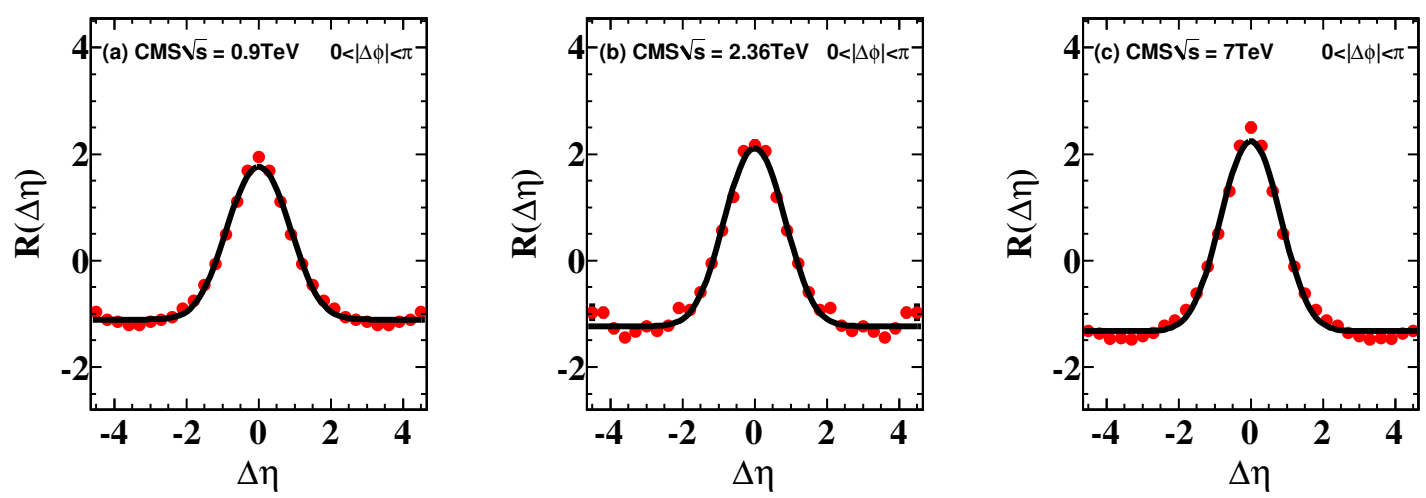

Figure 4. Two-particle pseudorapidity correlation function, obtained by averaging over the entire $\Delta \phi$ range from 0 to $\pi$, in $p p$ collisions at $\sqrt{s}=$ (a) 0.9 , (b) 2.36 , and (c) $7 \mathrm{TeV}$. The solid curves correspond to the fits by the cluster model using eq. (6.2). Error bars are smaller than the symbols.

where $\delta$ quantifies the average spread of particles originating from a single cluster, i.e. the decay width. The background distribution, $B(\Delta \eta)$, in eq. (6.2) is the same eventmixed distribution defined in eq. (4.3) but averaged over all the multiplicity bins with all corrections applied, and integrated over $\Delta \phi$.

Without knowing $\sigma_{K}$, the width of the distribution of $K$, it is impossible to calculate the average cluster size $\langle K\rangle$ directly from the measured value of $\alpha$. However, an effective cluster size can be defined using the extracted correlation strength via the relation:

$$
K_{\mathrm{eff}}=\alpha+1=\frac{\langle K(K-1)\rangle}{\langle K\rangle}+1=\langle K\rangle+\frac{\sigma_{K}^{2}}{\langle K\rangle} .
$$

The effective cluster size $K_{\text {eff }}$ and decay width $\delta$ can be estimated by means of a least $\chi^{2}$ fit of eq. (6.2) to the measured two-particle pseudorapidity correlation function. The ICM provides a good fit to the data over a large range in $\Delta \eta$, as shown in figure 4 .

The statistical uncertainties of the fit parameters are much smaller than the systematic ones. The correction for event selection efficiency (see section 5.2) has an overall systematic uncertainty of less than $2.8 \%$ found by comparing the result at the generator level to that from the reconstructed tracks after corrections. The model dependence of this procedure (i.e. the selection efficiency for NSD events) was investigated by using correction factors derived from different MC generators such as PYTHIA, PHOJET [29], and HERWIG++ [30]. The biggest discrepancy in the final results was about $2.6 \%$.

Systematic uncertainties due to track quality cuts were examined by loosening the cuts on the significance of both the transverse track impact parameter, $d_{x y} / \sigma\left(d_{x y}\right)$, and the distance along the beam to the primary vertex, $d_{z} / \sigma\left(d_{z}\right)$ from 3 to 5 . The final results were found to be insensitive to these track selections to within $1.2 \%$.

A summary of systematic uncertainties for the inclusive analysis is given in table 2 . The uncertainties are presented for the cluster model fit parameters listed in table 2, namely the correlation strength $\left(\alpha=K_{\text {eff }}-1\right)$ and the width in pseudorapidity $(\delta)$. 


\begin{tabular}{|lcc|}
\hline & \multicolumn{2}{c|}{ Systematic uncertainties [\%] } \\
\hline Source & $K_{\text {eff }}-1(\alpha)$ & $\delta$ \\
\hline Track quality cuts & 1.2 & 1.0 \\
Correction for tracking/acceptance efficiency and fake rate & 1.3 & 1.4 \\
Correction for event selection efficiency & 2.6 & 2.8 \\
Model dependence of the corrections & 2.6 & 1.3 \\
\hline Total systematic uncertainties & 4.1 & 3.5 \\
\hline
\end{tabular}

Table 2. Summary of systematic uncertainties in the inclusive analysis.

\begin{tabular}{|lcc|}
\hline$\sqrt{s}$ & $K_{\text {eff }}^{|\eta|<2.4}$ & $\delta^{|\eta|<2.4}$ \\
\hline $0.9 \mathrm{TeV}$ & $2.12 \pm<0.01$ (stat.) \pm 0.05 (syst.) & $0.53 \pm<0.01$ (stat.) \pm 0.02 (syst.) \\
$2.36 \mathrm{TeV}$ & $2.23 \pm 0.02$ (stat.) \pm 0.05 (syst.) & $0.52 \pm<0.01$ (stat.) \pm 0.02 (syst.) \\
$7 \mathrm{TeV}$ & $2.34 \pm<0.01$ (stat.) \pm 0.06 (syst.) & $0.51 \pm<0.01$ (stat.) \pm 0.02 (syst.) \\
\hline
\end{tabular}

Table 3. Final results on $K_{\text {eff }}$ and $\delta$ measured within the kinematic cuts of $p_{T}>0.1 \mathrm{GeV} / c$ and $|\eta|<2.4$ at CMS.

\begin{tabular}{|lrr|}
\hline$\sqrt{s}$ & \multicolumn{1}{|c|}{$K_{\text {eff }}^{|\eta|<3.0}$} & \multicolumn{1}{c|}{$\delta^{|\eta|<3.0}$} \\
\hline $0.9 \mathrm{TeV}$ & $2.50 \pm<0.01$ (stat.) \pm 0.06 (syst.) \pm 0.07 & $0.64 \pm<0.01$ (stat.) \pm 0.02 (syst.) \pm 0.03 \\
$2.36 \mathrm{TeV}$ & $2.65 \pm 0.03$ (stat.) \pm 0.07 (syst.) \pm 0.08 & $0.60 \pm 0.01$ (stat.) \pm 0.02 (syst.) \pm 0.03 \\
$7 \mathrm{TeV}$ & $2.75 \pm<0.01$ (stat.) \pm 0.07 (syst.) \pm 0.09 & $0.59 \pm<0.01$ (stat.) \pm 0.02 (syst.) \pm 0.03 \\
\hline
\end{tabular}

Table 4. Final results on $K_{\text {eff }}$ and $\delta$ measured by CMS after extrapolation to $p_{T}>0$ and $|\eta|<3$. The third quoted uncertainty is due to the extrapolation procedure.

Values of effective cluster sizes and widths observed within the kinematic cuts on $p_{T}$ and $|\eta|$ are summarized in table 3 .

As can be seen in figure 4 , the most central point of the 1-D pseudorapidity correlation function always lies slightly above the fits. This could be due to the residual effects of secondary processes that were not fully removed by the track selection, as well as BEC or other physics processes at this small scale in $\Delta \eta$. All fits exclude this central point, but including it in the fit affects the values of $K_{\text {eff }}$ and $\delta$ by no more than $0.5 \%$.

In figure 5, CMS measurements of $K_{\text {eff }}$ and $\delta$ for $p_{T}>0.1 \mathrm{GeV} / c$ and $|\eta|<2.4$ are shown as functions of $\sqrt{s}$, and compared with the PYTHIA D6T tune. An energy dependence of $K_{\text {eff }}$ is observed, while $\delta$ remains roughly constant over the three energies. PYTHIA shows energy dependencies of $K_{\text {eff }}$ and $\delta$ similar to those seen in the data, but the magnitude of $K_{\text {eff }}$ is significantly smaller in PYTHIA. The effect of tensor mesons in PYTHIA was investigated, but even using an unrealistically large probability of $50 \%$ for the angular momentum $\mathrm{L}=1$ meson states accounted for only about one third of the difference. Results from the HERWIG ++ model were also studied and found to have correlation function shapes dramatically different from the data, in agreement with previous results [31] showing that HERWIG++ is insufficiently tuned to reproduce soft QCD processes. 


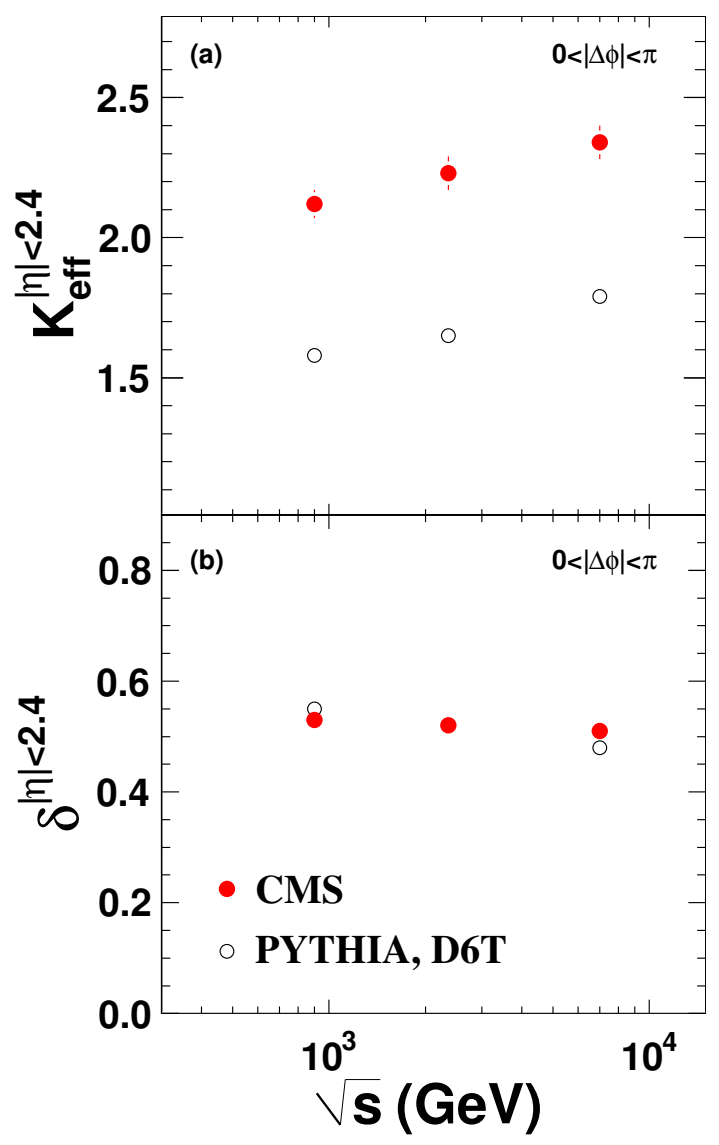

Figure 5. (a) $K_{\text {eff }}$ and (b) $\delta$ as a function of $\sqrt{s}$, measured for $p_{T}>0.1 \mathrm{GeV} / c$ and $|\eta|<2.4$ by CMS in solid circles. Open circles show the PYTHIA results with the D6T tune.

In order to compare with measurements made at lower energies, CMS results were extrapolated to $|\eta|<3$ and the full $p_{T}$ range $p_{T}>0$ to achieve a consistent kinematic range. The fraction of tracks below $p_{T} \approx 0.1 \mathrm{GeV} / c$ was estimated by fitting the measured $p_{T}$ distributions using the Tsallis function as was done in ref. [21], which empirically describes both the low $p_{T}$ exponential and the high $p_{T}$ power-law behaviors [32]. The integral of the fit function for $p_{T}<0.1 \mathrm{GeV} / c$ amounts to about $5.5 \%$ of the total yield, consistent with the results in ref. [21]. As first quantified in ref. [7], the loss of particles falling outside a limited $\eta$ acceptance results in a significant reduction of both $K_{\text {eff }}$ and $\delta$. This effect was investigated using several dynamical models as well as the simple ICM following the identical approach used in ref. [7]. As was the case in the previous analysis, the ratios of $K$ and $\delta$ for different $\eta$ acceptances $(|\eta|<3.0$ and $|\eta|<2.4$ in the present work) were found to scale very closely with $\delta^{|\eta|<2.4}$, the measured cluster width using data in the $|\eta|<2.4$ region, reinforcing the conclusion that the dependence of the extracted cluster parameters on pseudorapidity acceptance is primarily a simple geometric effect.

Figure 6 shows the results of $K_{\text {eff }}$ and $\delta$ measured by the CMS experiment after the extrapolation to $|\eta|<3$ and $p_{T} \approx 0$, as well as previous measurements at lower energies in the same pseudorapidity range $[3,5,6]$. Values of the extrapolated CMS results are sum- 


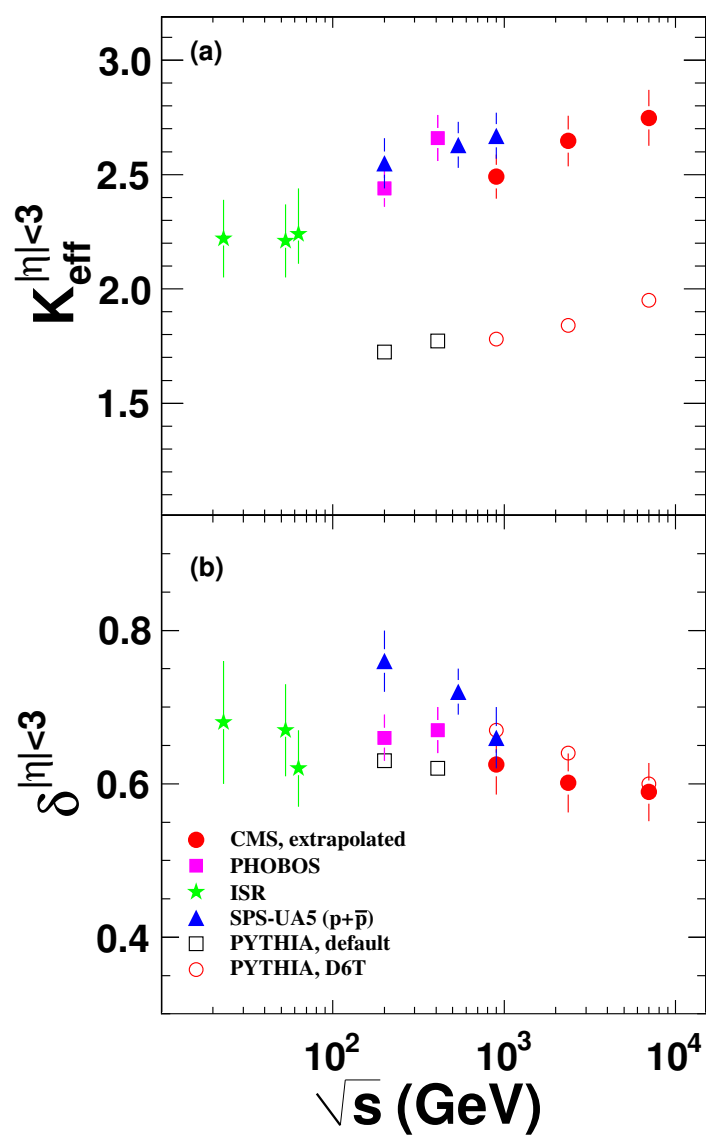

Figure 6. (a) $K_{\text {eff }}$ and (b) $\delta$ as a function of $\sqrt{s}$ based on a model-dependent extrapolation of CMS data to $p_{T} \approx 0$ and $|\eta|<3$ (solid circles), as well as data from PHOBOS [6] (solid squares), UA5 [5] (solid triangles) and ISR [3] (solid stars) experiments for $p p$ and $p \bar{p}$ collisions. Open circles and squares show the PYTHIA results for the D6T tune and default parameters, respectively.

marized in table 4, where the third quoted uncertainty is due to the extrapolation. The error bars in figure 6 include the systematic uncertainties from both the experimental measurements and the extrapolations added in quadrature. Events generated with PYTHIA D6T tune show a similar energy dependence of $K_{\text {eff }}$ and $\delta$ as the data, but systematically underestimate the magnitude of $K_{\text {eff }}$ over the full energy range.

The observed cluster size cannot be fully explained by a resonance decay model even at very low energies, since the expectation of $\langle K\rangle$ from resonance decays is about 1.5 (extrapolating to 1.7 for $K_{\text {eff }}$ depending on the assumed cluster size distribution [5]). This is significantly lower than the observed values, but is close to what is seen in PYTHIA. Additional sources of pseudorapidity correlations, such as local quantum number conservation [33], are needed to describe the data. As the energy increases further (especially at the $\mathrm{TeV}$ scale), the onset of jets should play a more important role in the particle production, resulting in bigger clusters. This effect could be the underlying cause for the observed energy dependence of $K_{\text {eff }}$. 


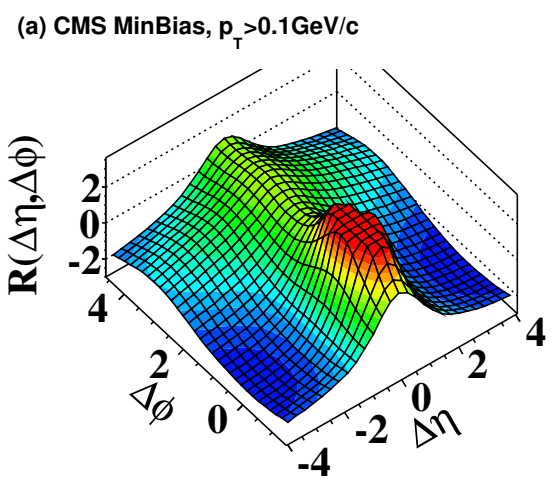

(b) CMS MinBias, $1.0 \mathrm{GeV} / \mathrm{c}<\mathrm{p}_{\mathrm{T}}<3.0 \mathrm{GeV} / \mathrm{c}$

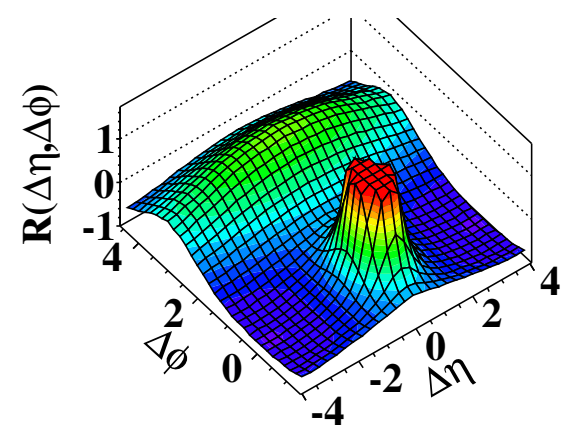

(c) CMS N $\geq 110, \mathrm{p}_{\mathrm{T}}>0.1 \mathrm{GeV} / \mathrm{c}$

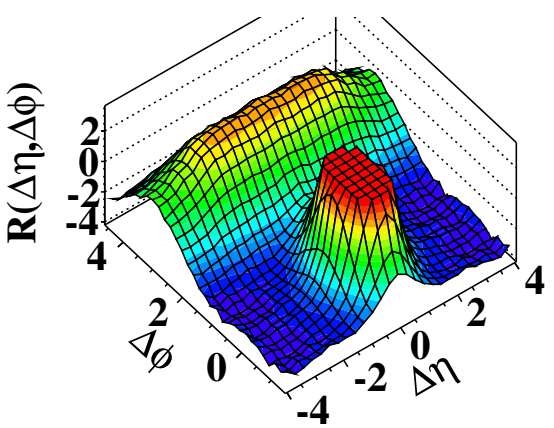

(d) $\mathrm{CMS} \mathrm{N} \geq 110,1.0 \mathrm{GeV} / \mathrm{c}<\mathrm{p}_{\mathrm{T}}<3.0 \mathrm{GeV} / \mathrm{c}$

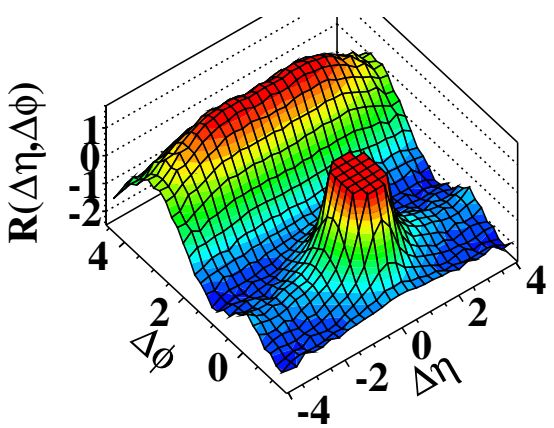

Figure 7. 2-D two-particle correlation functions for $7 \mathrm{TeV} p p$ (a) minimum bias events with $p_{T}>$ $0.1 \mathrm{GeV} / c$, (b) minimum bias events with $1<p_{T}<3 \mathrm{GeV} / c$, (c) high multiplicity $\left(N_{\mathrm{trk}}^{\text {offline }} \geq 110\right.$ ) events with $p_{T}>0.1 \mathrm{GeV} / c$ and (d) high multiplicity $\left(N_{\text {trk }}^{\text {offline }} \geq 110\right)$ events with $1<p_{T}<3 \mathrm{GeV} / c$. The sharp near-side peak from jet correlations is cut off in order to better illustrate the structure outside that region.

\section{Long-range correlations in $7 \mathrm{TeV}$ data}

The study of long-range azimuthal correlations involved generating 2-D $\Delta \eta-\Delta \phi$ distributions in bins of event multiplicity and particle transverse momentum. The analysis procedure was to a large extent identical with that used for the minimum bias data described in section 4 . With the addition of $p_{T}$ binning, both particles in the pairs used to calculate $R(\Delta \eta, \Delta \phi)$ were required to be within the selected $p_{T}$ range. The events were divided into bins of offline track multiplicity as outlined in table 1. In order to reach good statistics for the highest attainable charged particle densities, only data at $7 \mathrm{TeV}$ were considered.

Figure 7 compares 2-D two-particle correlation functions for minimum bias events and high multiplicity events, for both inclusive particles and for particles in an intermediate $p_{T}$ bin. The top two panels show results from minimum bias events. The correlation function for inclusive particles with $p_{T}>0.1 \mathrm{GeV} / c$ shows the typical structure as described by the independent cluster model. The region at $\Delta \eta \approx 0$ and intermediate $\Delta \phi$ is dominated 
by particle emission from clusters with low transverse momentum, with some contribution from jet-like particle production near $(\Delta \eta, \Delta \phi) \approx(0,0)$ due to near-side jet fragmentation and a broad elongated ridge around $\Delta \phi \approx \pi$ due to fragmentation of back-to-back jets. Also visible is a shallow minimum at $\Delta \phi \approx 0$ at large $|\Delta \eta|$ due to momentum conservation. For the intermediate $p_{\mathrm{T}}$ region of $1 \mathrm{GeV} / c<p_{T}<3 \mathrm{GeV} / c$ a more pronounced near-side jet peak and away-side ridge are visible, due to the enhanced contribution of jet fragmentation to particle production for increasing $p_{T}$.

For $p_{T}$-integrated two-particle correlations in high multiplicity events $\left(N_{\text {trk }}^{\text {offline }} \geq 110\right.$, figure $7 \mathrm{c}$ ), most correlation structures are similar to those for minimum bias events. The cut on high multiplicity enhances the relative contribution of high $p_{T}$ jets which fragment into a large number of particles and, therefore, has a qualitatively similar effect on the shape as the particle $p_{T}$ cut on minimum bias events (compare figure $7 \mathrm{~b}$ and figure $7 \mathrm{c}$ ). However, it is interesting to note that a closer inspection of the shallow minimum at $\Delta \phi \approx 0$ and $|\Delta \eta|>2$ in high multiplicity $p_{T}$-integrated events reveals it to be slightly less pronounced than that in minimum bias collisions.

Moving to the intermediate $p_{T}$ range in high multiplicity events shown in figure $7 \mathrm{~d}$, an unexpected effect is observed in the data. A clear and significant "ridge"-like structure emerges at $\Delta \phi \approx 0$ extending to $|\Delta \eta|$ of at least 4 units. This is a novel feature of the data which has never been seen in two-particle correlation functions in $p p$ or $p \bar{p}$ collisions. Simulations using MC models do not predict such an effect. An identical analysis of high multiplicity events in PYTHIA8 [34] results in correlation functions which do not exhibit the extended ridge at $\Delta \phi \approx 0$ seen in figure $7 \mathrm{~d}$, while all other structures of the correlation function are qualitatively reproduced. PYTHIA 8 was used to compare to these data since it produces more high multiplicity events than PYTHIA6 in the D6T tune. Several other PYTHIA tunes, as well as HERWIG++ [30] and Madgraph [35] events were also investigated. No evidence for near-side correlations corresponding to those seen in data was found.

The novel structure in the high multiplicity $p p$ data is reminiscent of correlations seen in relativistic heavy ion data. In the latter case, the observed long-range correlations are generally assumed to arise from various components of hydrodynamic flow of the produced medium [9, 36-39], from interactions between hard scattering processes and the medium, and from collective effects in the initial interaction of the nuclei.

However, new correlations can also start to emerge in the new energy regime probed here due to more elementary processes. For example, long range correlations are predicted also to occur in systems with a large number of fluctuating components, e.g. originating from additional color string connections. Such effects are presently not modeled in the MC generators.

Compared to the minimum bias analysis, the online and offline event selection of the rare high multiplicity events eliminated some sources of systematic uncertainties, but also introduced several additional ones. The bias due to the selection efficiency for NSD events, and its associated correction, were not an issue for the high multiplicity analysis since the efficiency reaches $100 \%$ as discussed in section 5.2. However, it was necessary to correct for the inefficiency in the HLT selection shown in figure 1. Comparison of correlation functions 
for the high multiplicity bin, $N_{\text {trk }}^{\text {offline }} \geq 110$, taken from the two different trigger paths, $\left(N_{\text {trk }}^{\text {online }}>70\right.$ and $N_{\text {trk }}^{\text {online }}>85$, see section 5.3$)$ showed a systematic variation of 4 to $5 \%$.

The pile-up rate (fraction of events with more than one good offline vertex found) reached about $40 \%$ for the LHC conditions pertaining for most of the high multiplicity data-taking. Studies on correlations between two offline vertices in each event showed that about $10 \%$ of the events contained pile-up that could not be distinguished by the vertex finding algorithm. Therefore, a high multiplicity event could be faked by a pileup of several minimum bias collisions with very close vertex positions. Although such pile-up of independent $p p$ collisions is not expected to generate additional correlations in this analysis, a data-driven limit on the effect of pile-up events was established. This was based on a comparison of results from runs with negligible pile-up collected with lower instantaneous luminosity to results obtained with high luminosity data at nominal bunch intensity. This comparison was limited by the size of the event sample collected for low luminosity conditions in early LHC running. For all luminosity selections, the near-side ridge signal was observed and a conservative systematic error of $15 \%$, which covers the difference over all run periods, was assigned.

In order to investigate the turn-on behavior of the "ridge"-like structure quantitatively and in finer detail, correlation functions were obtained in four bins of charged particle multiplicity and four bins of particle transverse momentum. To study the long-range azimuthal correlations, the 1-D $\Delta \phi$ were calculated by integrating over the $2.0<|\Delta \eta|<4.8$ region. Figure 8 shows the results for a range of $p_{T}$ (from left to right) and multiplicity (from top to bottom) bins. CMS data are shown as solid circles and the lines show PYTHIA8 results. In this projection, only the range of $0<\Delta \phi<\pi$ is shown, as the $\Delta \phi$ correlation function is symmetric around $\Delta \phi=0$ by construction. All panels show the away-side jet contribution at $\Delta \phi \approx \pi$. In addition, for high multiplicity bins in the intermediate $p_{\mathrm{T}}$ region, $1<p_{\mathrm{T}}<3 \mathrm{GeV} / c$, a second local maximum near $\Delta \phi \approx 0$ is clearly observed. This new feature of the long-range azimuthal correlation function is not present in low multiplicity or minimum bias data, which are dominated by the low multiplicity events.

The comparison of data to PYTHIA8 simulations is characterized by two discrepancies: the strength of the away-side correlation is over- or underpredicted for almost all bins. This quantitative discrepancy could be remedied by further tuning of the relative contributions of di-jet and multi-jet processes compared to particle production from soft processes in the model without introducing a qualitatively new mechanism. More importantly, PYTHIA8 qualitatively fails to reproduce the novel local maximum near $\Delta \phi \approx 0$ in any of the $p_{T}$ or multiplicity bins. It appears that soft particle production from string fragmentation, the contribution from jet fragmentation, final-state radiation, and concurrent semihard multiparton interactions, to the extent they are parametrized in PYTHIA8, do not provide a mechanism to create the observed long-range, near-side particle correlations.

Figure 8 shows that the long-range, near-side correlation increases in strength with increasing multiplicity and is most prominent in the region of $1<p_{\mathrm{T}}<3 \mathrm{GeV} / c$. The strength of the near-side ridge and its dependence on $p_{T}$ and multiplicity can be quantified in more detail by calculating the associated yield, i.e., the number of other particles correlated with a specific particle. In the presence of multiple sources of correlations, the 


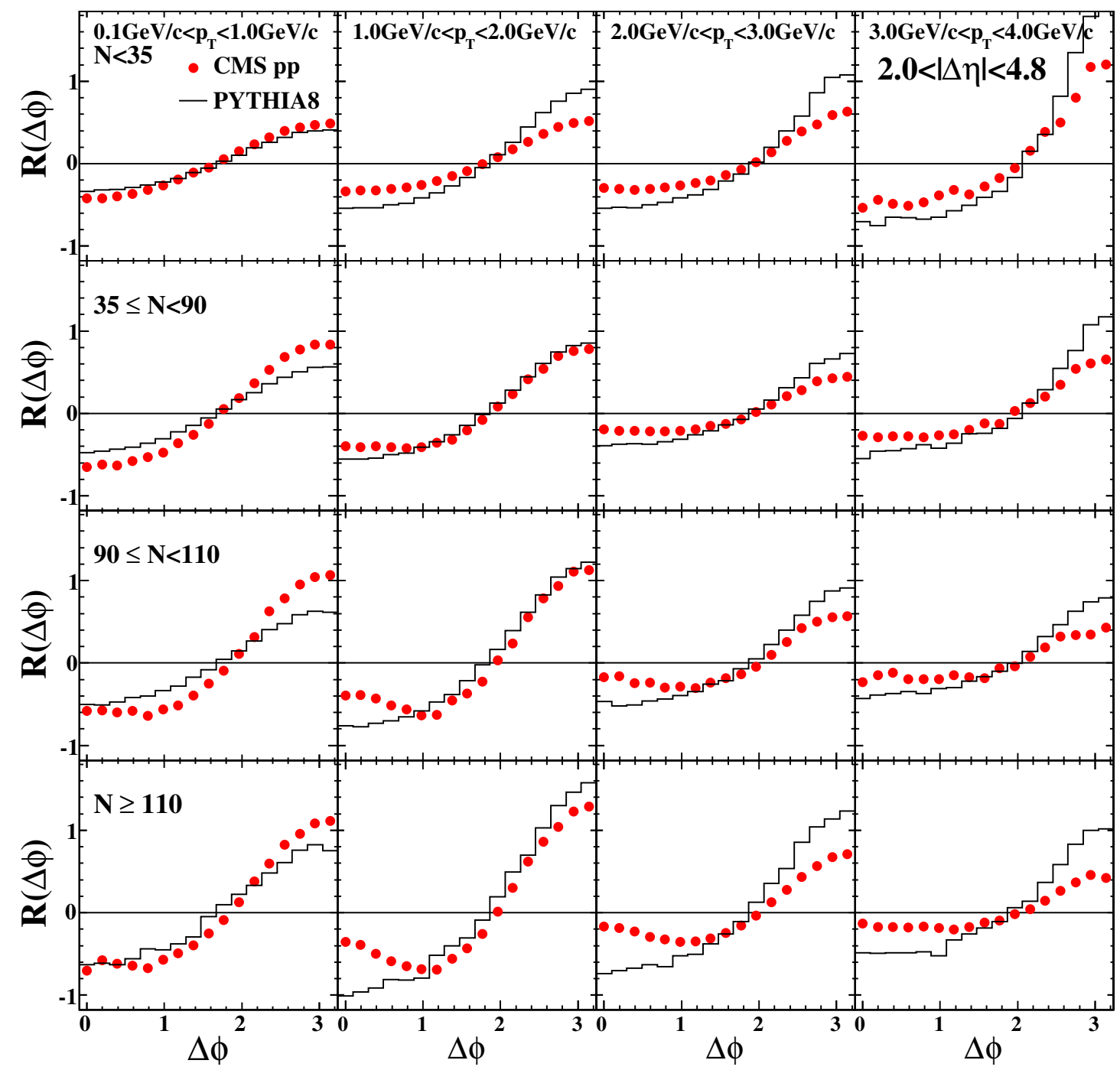

Figure 8. Projections of 2-D correlation functions onto $\Delta \phi$ for $2.0<|\Delta \eta|<4.8$ in different $p_{T}$ and multiplicity bins for fully corrected $7 \mathrm{TeV} p p$ data and reconstructed PYTHIA 8 simulations. Error bars are smaller than the symbols.

yield for the correlation of interest is commonly estimated using an implementation of the zero-yield-at-minimum (ZYAM) method [40]. The procedure uses $\mathrm{R}(\Delta \phi)$ integrated over $2.0<|\Delta \eta|<4.8$ as shown in figure 8. In the first step, a second-order polynomial is fit to $\mathrm{R}(\Delta \phi)$ in the region $0.1<|\Delta \phi|<2.0$. The location of the minimum of the polynomial in this region is denoted $\Delta \phi_{\mathrm{ZYAM}}$. The contribution from the background source of correlations, in this case the away-side jet correlations, is assumed to be zero for $|\Delta \phi| \leq \Delta \phi_{\mathrm{ZYAM}}$. Using the position of the minimum, the associated yield is then found by integrating $R(\Delta \phi)$ over the region $0<|\Delta \phi|<\Delta \phi_{\text {ZYAM }}$ relative to the minimum in $\mathrm{R}(\Delta \phi)$ and multiplying by $\int_{2.0}^{4.8} \mathrm{~B}(\Delta \eta) \mathrm{d}|\Delta \eta|$ to account for the fact that only a limited $\Delta \eta$ range is used. The uncertainty on the minimum level of $R(\Delta \phi)$ obtained by the ZYAM procedure as well as varying the fit range in $\Delta \phi$ gives an uncertainty of 0.0025 on the associated yield, uniformly over all multiplicity and $p_{T}$ bins. 


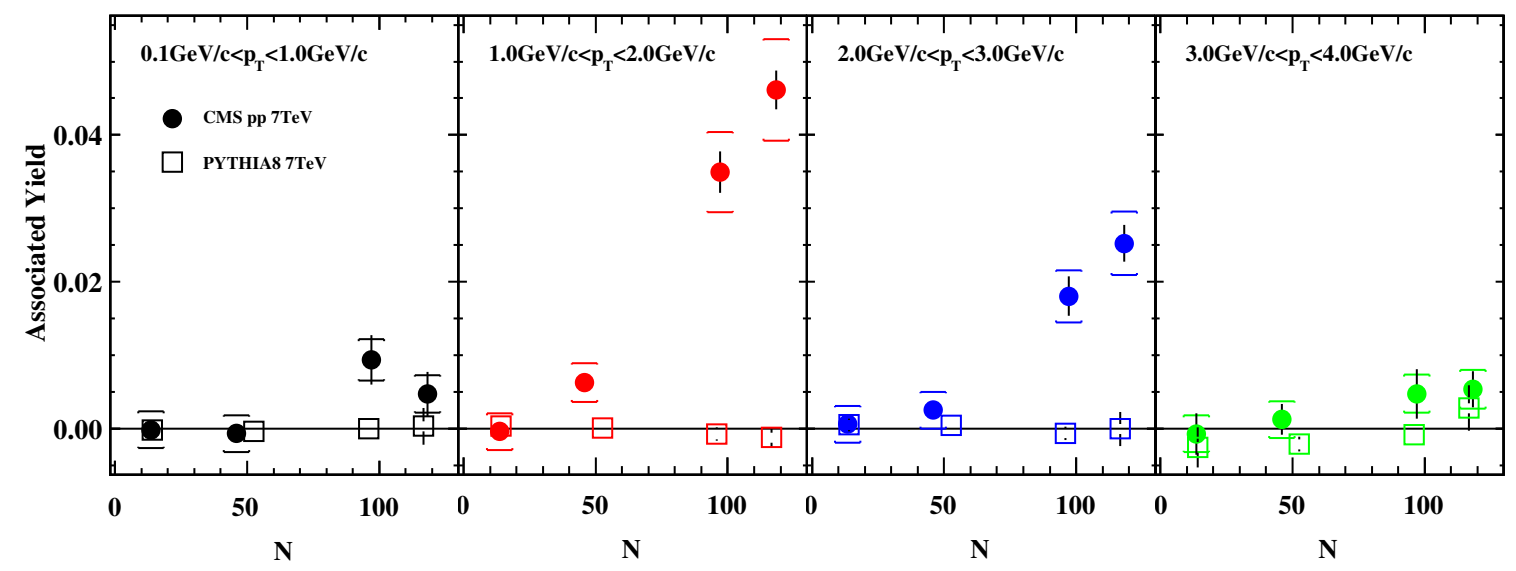

Figure 9. Associated yield for the near-side of the correlation function integrated over the region of $2.0<|\Delta \eta|<4.8$ as a function of event multiplicity in bins of $p_{T}$ for $7 \mathrm{TeV} p p$ collisions. The error bars correspond to statistical errors, while the brackets around the data points denote the systematic uncertainties. The open squares show results for PYTHIA8.

Figure 9 shows the associated yield as a function of event multiplicity integrated over $2.0<|\Delta \eta|<4.8$ in increasing bins of $p_{T}$. The ridge yield is consistent with zero for low multiplicity events. The emergence of the ridge is observed toward the very high multiplicity region, primarily for the intermediate $p_{T}$ range of $1-3 \mathrm{GeV} / c$. The error bars correspond to statistical errors, while the brackets around the data points denote the systematic uncertainties. Results from the PYTHIA8 MC, shown in open squares in figure 9 , are consistent with zero for all multiplicity and $p_{T}$ regions, indicating that the ridge observed in the data is totally absent in events produced by this generator.

To investigate the novel ridge-like structure further, two-particle correlations were calculated separately for like-sign and unlike-sign charged pairs. Possible problems related to the track reconstruction algorithm, like multiple reconstruction of the same particle or local occupancy changes, would be expected to affect like-sign pairs differently than unlike-sign pairs. The same choice of pairs of like- or unlike-sign was made for both the signal and background in eq. (4.1). Figure 10 shows the associated yield for like-sign (solid circles) and unlike-sign (open squares) two-particle correlations respectively as a function of event multiplicity integrated over $2.0<|\Delta \eta|<4.8$ in bins of $p_{T}$. Consistent multiplicity and $p_{\mathrm{T}}$ dependencies of the near-side associated yield are observed for charge dependent and charge independent correlations. The results for like-sign and unlike-sign pairs agree with each other within uncertainties. Since the number of like- and unlike-sign pairs each represent roughly half of the total, the yield of associated pairs counting only one sign option is expected to be roughly a factor of two smaller than the unrestricted yield.

As a further cross-check, correlation functions were generated for tracks paired with ECAL photons (primarily due to $\pi^{0} \mathrm{~s}$ ) as well as pairs of two ECAL photons. These distributions showed similar behavior to those shown in figures 7 and 8, i.e., the high $|\Delta \eta|$ region contained a dip at $|\Delta \phi| \approx 0$ in minimum bias events and a ridge in that region for high multiplicity events. Data at 0.9 and $2.36 \mathrm{TeV}$ were also analyzed for long-range correlations, but the statistics were not sufficient to draw a conclusion. 


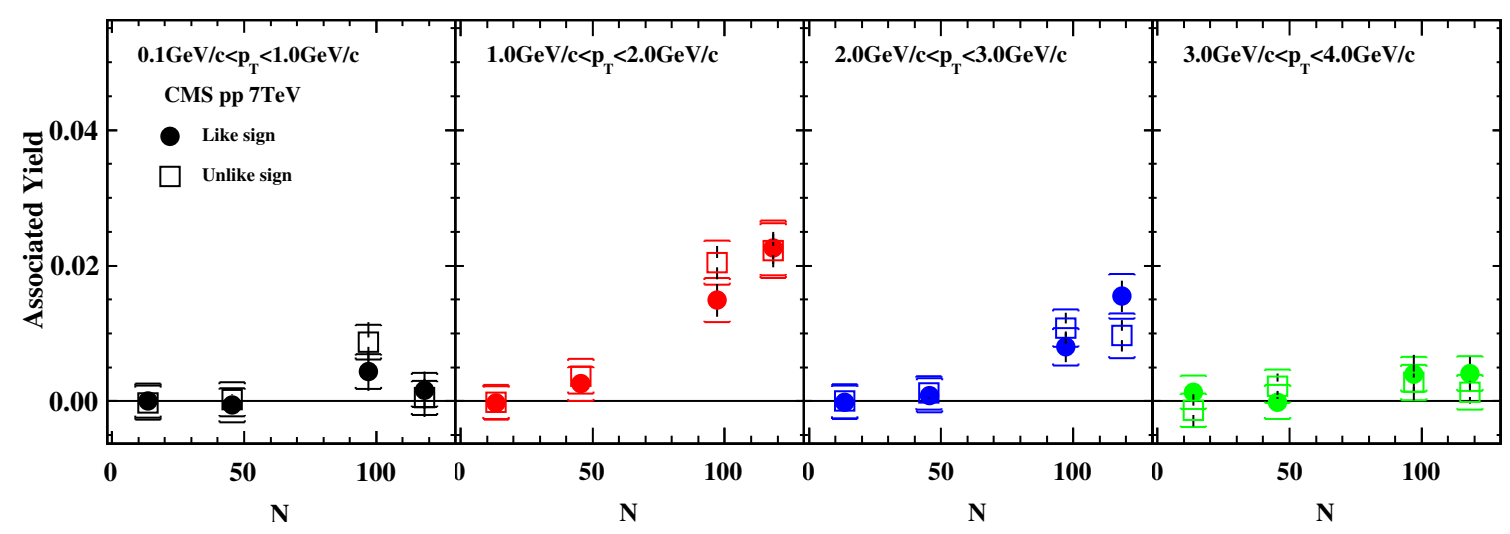

Figure 10. Like-sign and unlike-sign associated yield for the near-side of the correlation function integrated over the region of $2.0<|\Delta \eta|<4.8$ as a function of event multiplicity in bins of $p_{T}$. The error bars correspond to statistical errors, while the brackets around the data points denote the systematic uncertainties.

\section{Conclusion}

The CMS detector at the LHC has been used to measure angular correlations between two charged particles up to $|\Delta \eta| \approx 5$ and over the full range of $\Delta \phi$ in $p p$ collisions at $\sqrt{s}=$ $0.9,2.36$, and $7 \mathrm{TeV}$. The extracted $2-\mathrm{D}$ correlation functions show a variety of features. In minimum bias collisions they are dominated by a maximum at $\Delta \eta=0$ extending over the full range in $\Delta \phi$, with a width which tends to increase with increasing $\Delta \phi$. A simple cluster model parametrization was fit to these short-range correlations in order to quantify their strength (the effective cluster size) and their extent in relative pseudorapidity (the cluster decay width). The cluster size is observed to increase slowly with beam energy, while the cluster width is essentially constant. The PYTHIA event generator with D6T tune correctly describes the cluster widths and the energy dependence of the cluster size but systematically underestimates the cluster size.

Long-range azimuthal correlations for $2.0<|\Delta \eta|<4.8$ have been studied for $7 \mathrm{TeV}$ data, leading to the first observation of a long-range ridge-like structure at the near-side $(\Delta \phi \approx 0)$ in $p p$ collisions. This striking feature is clearly seen for large rapidity differences $|\Delta \eta|>2$ in events with an observed charged particle multiplicity of $N \approx 90$ or higher. The enhancement in the near-side correlation function is most evident in the intermediate transverse momentum range, $1<p_{T}<3 \mathrm{GeV} / c$. In the $2.0<|\Delta \eta|<4.8$ range, a steep increase of the near-side associated yield with multiplicity has been found in the data, whereas simulations show an associated yield consistent with zero, independent of multiplicity and transverse momentum. The novel structure resembles similar features observed in heavy ion experiments [7-9]. However, the physical origin of our observation is not yet understood. Additional characteristics of the high multiplicity $p p$ events displaying this novel feature deserve further detailed study. 


\section{Acknowledgments}

We thank the technical and administrative staff at CERN and other CMS institutes. This work was supported by the Austrian Federal Ministry of Science and Research; the Belgium Fonds de la Recherche Scientifique, and Fonds voor Wetenschappelijk Onderzoek; the Brazilian Funding Agencies (CNPq, CAPES, FAPERJ, and FAPESP); the Bulgarian Ministry of Education and Science; CERN; the Chinese Academy of Sciences, Ministry of Science and Technology, and National Natural Science Foundation of China; the Colombian Funding Agency (COLCIENCIAS); the Croatian Ministry of Science, Education and Sport; the Research Promotion Foundation, Cyprus; the Estonian Academy of Sciences and NICPB; the Academy of Finland, Finnish Ministry of Education, and Helsinki Institute of Physics; the Institut National de Physique Nucléaire et de Physique des Particules / CNRS, and Commissariat à l'Énergie Atomique, France; the Bundesministerium für Bildung und Forschung, Deutsche Forschungsgemeinschaft, and Helmholtz-Gemeinschaft Deutscher Forschungszentren, Germany; the General Secretariat for Research and Technology, Greece; the National Scientific Research Foundation, and National Office for Research and Technology, Hungary; the Department of Atomic Energy, and Department of Science and Technology, India; the Institute for Studies in Theoretical Physics and Mathematics, Iran; the Science Foundation, Ireland; the Istituto Nazionale di Fisica Nucleare, Italy; the Korean Ministry of Education, Science and Technology and the World Class University program of NRF, Korea; the Lithuanian Academy of Sciences; the Mexican Funding Agencies (CINVESTAV, CONACYT, SEP, and UASLP-FAI); the Pakistan Atomic Energy Commission; the State Commission for Scientific Research, Poland; the Fundação para a Ciência e a Tecnologia, Portugal; JINR (Armenia, Belarus, Georgia, Ukraine, Uzbekistan); the Ministry of Science and Technologies of the Russian Federation, and Russian Ministry of Atomic Energy; the Ministry of Science and Technological Development of Serbia; the Ministerio de Ciencia e Innovación, and Programa Consolider-Ingenio 2010, Spain; the Swiss Funding Agencies (ETH Board, ETH Zurich, PSI, SNF, UniZH, Canton Zurich, and SER); the National Science Council, Taipei; the Scientific and Technical Research Council of Turkey, and Turkish Atomic Energy Authority; the Science and Technology Facilities Council, UK; the US Department of Energy, and the US National Science Foundation.

Individuals have received support from the Marie-Curie IEF program (European Union); the Leventis Foundation; the A. P. Sloan Foundation; the Alexander von Humboldt Foundation; and the Associazione per lo Sviluppo Scientifico e Tecnologico del Piemonte (Italy).

Open Access. This article is distributed under the terms of the Creative Commons Attribution Noncommercial License which permits any noncommercial use, distribution, and reproduction in any medium, provided the original author(s) and source are credited.

\section{References}

[1] E.L. Berger, Rapidity Correlations at Fixed Multiplicity in Cluster Emission Models, Nucl. Phys. B 85 (1975) 61 [SPIRES].

[2] A. Morel and G. Plaut, How Do Clusters Look in Semiinclusive Cross-Sections?, Nucl. Phys. B 78 (1974) 541 [SPIRES]. 
[3] K. Eggert et al., Angular Correlations Between the Charged Particles Produced in pp Collisions at ISR Energies, Nucl. Phys. B 86 (1975) 201 [SPIRES].

[4] L. Foà, Inclusive Study of High-Energy Multiparticle Production and Two-Body Correlations, Phys. Rept. 22 (1975) 1 [SPIRES].

[5] UA5 collaboration, R.E. Ansorge et al., Charged particle correlations in $\bar{p} p$ collisions at C.M. energies of $200 \mathrm{GeV}, 546 \mathrm{GeV}$ and $900 \mathrm{GeV}, \mathrm{Z}$. Phys. C 37 (1988) 191 [SPIRES].

[6] PHOBOS collaboration, B. Alver et al., Cluster properties from two-particle angular correlations in pp collisions at $\sqrt{s}=200 \mathrm{GeV}$ and $410 \mathrm{GeV}$, Phys. Rev. C 75 (2007) 054913 [arXiv: 0704. 0966] [SPIRES].

[7] PHOBOS collaboration, B. Alver et al., System size dependence of cluster properties from two-particle angular correlations in $\mathrm{Cu}+\mathrm{Cu}$ and $\mathrm{Au}+\mathrm{Au}$ collisions at $\sqrt{s_{N N}}=200 \mathrm{GeV}$, Phys. Rev. C 81 (2010) 024904 [arXiv:0812.1172] [SPIRES].

[8] PHOBOS collaboration, B. Alver et al., High transverse momentum triggered correlations over a large pseudorapidity acceptance in Au+Au collisions at $\sqrt{s_{N N}}=200 \mathrm{GeV}$, Phys. Rev. Lett. 104 (2010) 062301 [arXiv: 0903.2811] [SPIRES].

[9] STAR collaboration, B.I. Abelev et al., Three-particle coincidence of the long range pseudorapidity correlation in high energy nucleus-nucleus collisions, Phys. Rev. Lett. 105 (2010) 022301 [arXiv:0912.3977] [SPIRES].

[10] B. Alver et al., System Size, Energy and Centrality Dependence of Pseudorapidity Distributions of Charged Particles in Relativistic Heavy Ion Collisions, Phys. Rev. Lett. 102 (2009) 142301 [arXiv:0709.4008] [SPIRES].

[11] L. Cunqueiro, J. Dias de Deus and C. Pajares, Nuclear like effects in proton-proton collisions at high energy, Eur. Phys. J. C 65 (2010) 423 [arXiv:0806.0523] [SPIRES].

[12] M. Luzum and P. Romatschke, Viscous Hydrodynamic Predictions for Nuclear Collisions at the LHC, Phys. Rev. Lett. 103 (2009) 262302 [arXiv:0901.4588] [SPIRES].

[13] I. Bautista, L. Cunqueiro, J.D. de Deus and C. Pajares, Particle production azimuthal asymmetries in a clustering of color sources, J. Phys. G 37 (2010) 015103 [arXiv: 0905.3058] [SPIRES].

[14] D. d'Enterria et al., Estimates of hadron azimuthal anisotropy from multiparton interactions in proton-proton collisions at $\sqrt{s}=14 \mathrm{TeV}$, Eur. Phys. J. C 66 (2010) 173 [arXiv:0910.3029] [SPIRES].

[15] S.K. Prasad, V. Roy, S. Chattopadhyay and A.K. Chaudhuri, Elliptic flow $\left(v_{2}\right)$ in pp collisions at LHC energy: a hydrodynamical approach, Phys. Rev. C 82 (2010) 024909 [arXiv: 0910 . 4844] [SPIRES].

[16] P. Bozek, Observation of the collective flow in proton-proton collisions, Acta Phys. Pol. B 41 (2010) 837 [arXiv: 0911.2392] [SPIRES].

[17] J. Casalderrey-Solana and U.A. Wiedemann, Eccentricity fluctuations make flow measurable in high multiplicity pp collisions, Phys. Rev. Lett. 104 (2010) 102301 [arXiv:0911.4400] [SPIRES].

[18] G. Ortona, G.S. Denicol, P. Mota and T. Kodama, Elliptic flow in high multiplicity proton-proton collisions at $\sqrt{s}=14 \mathrm{TeV}$ as a signature of deconfinement and quantum energy density fluctuations, arXiv:0911.5158 [SPIRES].

[19] CMS collaboration, The CMS Experiment at the CERN LHC, 2008 JINST 3 S08004.

[20] GEANT4 collaboration, S. Agostinelli et al., GEANT4: A simulation toolkit, Nucl. Instrum. Meth. A 506 (2003) 250 [SPIRES].

[21] CMS collaboration, V. Khachatryan et al., Transverse momentum and pseudorapidity distributions of charged hadrons in pp collisions at $\sqrt{s}=0.9$ and 2.36 TeV, JHEP 02 (2010) 041 [arXiv: 1002.0621] [SPIRES]. 
[22] CMS collaboration, V. Khachatryan et al., CMS Tracking Performance Results from early LHC Operation, arXiv: 1007.1988 [SPIRES].

[23] CMS collaboration, Measurement of Tracking Efficiency, CMS PAS TRK-10-002 (2010).

[24] Proceedings of $1^{\text {st }}$ workshop of Multiple Parton Interactions at the LHC, Perugia, Italy, October 27-31, 2008 [DESY-PROC-2009-06].

[25] M.A. Lisa and S. Pratt, Femtoscopically Probing the Freeze-out Configuration in Heavy Ion Collisions, arXiv:0811.1352 [SPIRES].

[26] UA1 collaboration, C. Albajar et al., Bose-Einstein correlations in $\bar{p} p$ interactions at $\sqrt{s}=0.2 \mathrm{TeV}$ to $0.9 \mathrm{TeV}$, Phys. Lett. B 226 (1989) 410 [SPIRES].

[27] CMS collaboration, V. Khachatryan et al., First Measurement of Bose-Einstein Correlations in Proton-Proton Collisions at $\sqrt{s}=0.9$ and $2.36 \mathrm{TeV}$ at the LHC, Phys. Rev. Lett. 105 (2010) 032001 [arXiv: 1005.3294] [SPIRES].

[28] ALICE collaboration, K. Aamodt et al., Two-pion Bose-Einstein correlations in pp collisions at $\sqrt{s}=900 \mathrm{GeV}$, arXiv: 1007.0516 [SPIRES].

[29] F.W. Bopp, R. Engel and J. Ranft, Rapidity gaps and the PHOJET Monte Carlo, hep-ph/9803437 [SPIRES].

[30] M. Bahr et al., HERWIG++ Physics and Manual, Eur. Phys. J. C 58 (2008) 639 [arXiv: 0803.0883] [SPIRES].

[31] DELPHI collaboration, J. Abdallah et al., Rapidity-alignment and $p_{T}$ compensation of particle pairs in hadronic $Z^{0}$ decays, Phys. Lett. B 533 (2002) 243 [hep-ex/0204038] [SPIRES].

[32] C. Tsallis, Possible Generalization of Boltzmann-Gibbs Statistics, J. Stat. Phys. 52 (1988) 479 [SPIRES].

[33] STAR collaboration, R.J. Porter and T.A. Trainor, Correlation structures from soft and semi-hard components in pp collisions at $\sqrt{s}=200 \mathrm{GeV}$, Acta Phys. Polon. B 36 (2005) 353 [SPIRES].

[34] T. Sjöstrand, S. Mrenna and P.Z. Skands, A Brief Introduction to PYTHIA 8.1, Comput. Phys. Commun. 178 (2008) 852 [arXiv:0710.3820] [SPIRES].

[35] J. Alwall et al., MadGraph/MadEvent v4: The New Web Generation, JHEP 09 (2007) 028 [arXiv:0706.2334] [SPIRES].

[36] P.F. Kolb, P. Huovinen, U.W. Heinz and H. Heiselberg, Elliptic flow at SPS and RHIC: From kinetic transport to hydrodynamics, Phys. Lett. B 500 (2001) 232 [hep-ph/0012137] [SPIRES].

[37] B.B. Back et al., The PHOBOS perspective on discoveries at RHIC, Nucl. Phys. A 757 (2005) 28 [nucl-ex/0410022] [SPIRES].

[38] STAR collaboration, J. Adams et al., Experimental and theoretical challenges in the search for the quark gluon plasma: The STAR collaboration's critical assessment of the evidence from RHIC collisions, Nucl. Phys. A 757 (2005) 102 [nucl-ex/0501009] [SPIRES].

[39] PHENIX collaboration, K. Adcox et al., Formation of dense partonic matter in relativistic nucleus nucleus collisions at RHIC: Experimental evaluation by the PHENIX collaboration, Nucl. Phys. A 757 (2005) 184 [nucl-ex/0410003] [SPIRES].

[40] N.N. Ajitanand et al., Decomposition of harmonic and jet contributions to particle-pair correlations at ultra-relativistic energies, Phys. Rev. C 72 (2005) 011902 [nucl-ex/0501025] [SPIRES]. 


\section{The CMS collaboration}

Yerevan Physics Institute, Yerevan, Armenia

V. Khachatryan, A.M. Sirunyan, A. Tumasyan

Institut für Hochenergiephysik der OeAW, Wien, Austria

W. Adam, T. Bergauer, M. Dragicevic, J. Erö, C. Fabjan, M. Friedl, R. Frühwirth, V.M. Ghete, J. Hammer ${ }^{1}$, S. Hänsel, C. Hartl, M. Hoch, N. Hörmann, J. Hrubec, M. Jeitler, G. Kasieczka, W. Kiesenhofer, M. Krammer, D. Liko, I. Mikulec, M. Pernicka, H. Rohringer, R. Schöfbeck, J. Strauss, A. Taurok, F. Teischinger, W. Waltenberger, G. Walzel, E. Widl, C.-E. Wulz

National Centre for Particle and High Energy Physics, Minsk, Belarus

V. Mossolov, N. Shumeiko, J. Suarez Gonzalez

Universiteit Antwerpen, Antwerpen, Belgium

L. Benucci, L. Ceard, E.A. De Wolf, X. Janssen, T. Maes, L. Mucibello, S. Ochesanu, B. Roland, R. Rougny, M. Selvaggi, H. Van Haevermaet, P. Van Mechelen, N. Van Remortel

Vrije Universiteit Brussel, Brussel, Belgium

V. Adler, S. Beauceron, S. Blyweert, J. D'Hondt, O. Devroede, A. Kalogeropoulos, J. Maes, M. Maes, S. Tavernier, W. Van Doninck, P. Van Mulders, I. Villella

Université Libre de Bruxelles, Bruxelles, Belgium

E.C. Chabert, O. Charaf, B. Clerbaux, G. De Lentdecker, V. Dero, A.P.R. Gay, G.H. Hammad, T. Hreus, P.E. Marage, C. Vander Velde, P. Vanlaer, J. Wickens

Ghent University, Ghent, Belgium

S. Costantini, M. Grunewald, B. Klein, A. Marinov, D. Ryckbosch, F. Thyssen, M. Tytgat, L. Vanelderen, P. Verwilligen, S. Walsh, N. Zaganidis

Université Catholique de Louvain, Louvain-la-Neuve, Belgium

S. Basegmez, G. Bruno, J. Caudron, J. De Favereau De Jeneret, C. Delaere, P. Demin,

D. Favart, A. Giammanco, G. Grégoire, J. Hollar, V. Lemaitre, O. Militaru, S. Ovyn,

D. Pagano, A. Pin, K. Piotrzkowski ${ }^{1}$, L. Quertenmont, N. Schul

Université de Mons, Mons, Belgium

N. Beliy, T. Caebergs, E. Daubie

Centro Brasileiro de Pesquisas Fisicas, Rio de Janeiro, Brazil

G.A. Alves, D. De Jesus Damiao, M.E. Pol, M.H.G. Souza

Universidade do Estado do Rio de Janeiro, Rio de Janeiro, Brazil

W. Carvalho, E.M. Da Costa, C. De Oliveira Martins, S. Fonseca De Souza, L. Mundim, H. Nogima, V. Oguri, J.M. Otalora Goicochea, W.L. Prado Da Silva, A. Santoro, S.M. Silva Do Amaral, A. Sznajder, F. Torres Da Silva De Araujo

Instituto de Fisica Teorica, Universidade Estadual Paulista, Sao Paulo, Brazil F.A. Dias, M.A.F. Dias, T.R. Fernandez Perez Tomei, E. M. Gregores ${ }^{2}$, F. Marinho, S.F. Novaes, Sandra S. Padula 
Institute for Nuclear Research and Nuclear Energy, Sofia, Bulgaria

N. Darmenov ${ }^{1}$, L. Dimitrov, V. Genchev ${ }^{1}$, P. Iaydjiev $^{1}$, S. Piperov, M. Rodozov, S. Stoykova, G. Sultanov, V. Tcholakov, R. Trayanov, I. Vankov

University of Sofia, Sofia, Bulgaria

M. Dyulendarova, R. Hadjiiska, V. Kozhuharov, L. Litov, E. Marinova, M. Mateev, B. Pavlov, P. Petkov

Institute of High Energy Physics, Beijing, China

J.G. Bian, G.M. Chen, H.S. Chen, C.H. Jiang, D. Liang, S. Liang, J. Wang, J. Wang, X. Wang, Z. Wang, M. Yang, J. Zang, Z. Zhang

State Key Lab. of Nucl. Phys. and Tech., Peking University, Beijing, China Y. Ban, S. Guo, Z. Hu, W. Li, Y. Mao, S.J. Qian, H. Teng, B. Zhu

Universidad de Los Andes, Bogota, Colombia

A. Cabrera, B. Gomez Moreno, A.A. Ocampo Rios, A.F. Osorio Oliveros, J.C. Sanabria

Technical University of Split, Split, Croatia

N. Godinovic, D. Lelas, K. Lelas, R. Plestina ${ }^{3}$, D. Polic, I. Puljak

University of Split, Split, Croatia

Z. Antunovic, M. Dzelalija

Institute Rudjer Boskovic, Zagreb, Croatia

V. Brigljevic, S. Duric, K. Kadija, S. Morovic

University of Cyprus, Nicosia, Cyprus

A. Attikis, R. Fereos, M. Galanti, J. Mousa, C. Nicolaou, F. Ptochos, P.A. Razis, H. Rykaczewski

Academy of Scientific Research and Technology of the Arab Republic of Egypt, Egyptian Network of High Energy Physics, Cairo, Egypt

Y. Assran ${ }^{4}$, M.A. Mahmoud ${ }^{5}$

National Institute of Chemical Physics and Biophysics, Tallinn, Estonia

A. Hektor, M. Kadastik, K. Kannike, M. Müntel, M. Raidal, L. Rebane

Department of Physics, University of Helsinki, Helsinki, Finland

V. Azzolini, P. Eerola

Helsinki Institute of Physics, Helsinki, Finland

S. Czellar, J. Härkönen, A. Heikkinen, V. Karimäki, R. Kinnunen, J. Klem, M.J. Kortelainen, T. Lampén, K. Lassila-Perini, S. Lehti, T. Lindén, P. Luukka, T. Mäenpää, E. Tuominen, J. Tuominiemi, E. Tuovinen, D. Ungaro, L. Wendland

Lappeenranta University of Technology, Lappeenranta, Finland

K. Banzuzi, A. Korpela, T. Tuuva 
Laboratoire d'Annecy-le-Vieux de Physique des Particules, IN2P3-CNRS, Annecy-le-Vieux, France

D. Sillou

\section{DSM/IRFU, CEA/Saclay, Gif-sur-Yvette, France}

M. Besancon, M. Dejardin, D. Denegri, J. Descamps, B. Fabbro, J.L. Faure, F. Ferri, S. Ganjour, F.X. Gentit, A. Givernaud, P. Gras, G. Hamel de Monchenault, P. Jarry, E. Locci, J. Malcles, M. Marionneau, L. Millischer, J. Rander, A. Rosowsky, D. Rousseau, M. Titov, P. Verrecchia

Laboratoire Leprince-Ringuet, Ecole Polytechnique, IN2P3-CNRS, Palaiseau, France

S. Baffioni, L. Bianchini, M. Bluj ${ }^{6}$, C. Broutin, P. Busson, C. Charlot, L. Dobrzynski, R. Granier de Cassagnac, M. Haguenauer, P. Miné, C. Mironov, C. Ochando, P. Paganini, D. Sabes, R. Salerno, Y. Sirois, C. Thiebaux, A. Zabi

Institut Pluridisciplinaire Hubert Curien, Université de Strasbourg, Université de Haute Alsace Mulhouse, CNRS/IN2P3, Strasbourg, France

J.-L. Agram ${ }^{7}$, A. Besson, D. Bloch, D. Bodin, J.-M. Brom, M. Cardaci, E. Conte ${ }^{7}$, F. Drouhin ${ }^{7}$, C. Ferro, J.-C. Fontaine ${ }^{7}$, D. Gelé, U. Goerlach, S. Greder, P. Juillot, M. Karim ${ }^{7}$, A.-C. Le Bihan, Y. Mikami, P. Van Hove

Centre de Calcul de l'Institut National de Physique Nucleaire et de Physique des Particules (IN2P3), Villeurbanne, France

F. Fassi, D. Mercier

Université de Lyon, Université Claude Bernard Lyon 1, CNRS-IN2P3, Institut de Physique Nucléaire de Lyon, Villeurbanne, France

C. Baty, N. Beaupere, M. Bedjidian, O. Bondu, G. Boudoul, D. Boumediene, H. Brun,

N. Chanon, R. Chierici, D. Contardo, P. Depasse, H. El Mamouni, A. Falkiewicz, J. Fay,

S. Gascon, B. Ille, T. Kurca, T. Le Grand, M. Lethuillier, L. Mirabito, S. Perries, V. Sordini,

S. Tosi, Y. Tschudi, P. Verdier, H. Xiao

E. Andronikashvili Institute of Physics, Academy of Science, Tbilisi, Georgia

V. Roinishvili

RWTH Aachen University, I. Physikalisches Institut, Aachen, Germany

G. Anagnostou, M. Edelhoff, L. Feld, N. Heracleous, O. Hindrichs, R. Jussen, K. Klein, J. Merz, N. Mohr, A. Ostapchuk, A. Perieanu, F. Raupach, J. Sammet, S. Schael, D. Sprenger, H. Weber, M. Weber, B. Wittmer

RWTH Aachen University, III. Physikalisches Institut A, Aachen, Germany M. Ata, W. Bender, M. Erdmann, J. Frangenheim, T. Hebbeker, A. Hinzmann, K. Hoepfner, C. Hof, T. Klimkovich, D. Klingebiel, P. Kreuzer ${ }^{1}$, D. Lanske ${ }^{\dagger}$, C. Magass, G. Masetti, M. Merschmeyer, A. Meyer, P. Papacz, H. Pieta, H. Reithler, S.A. Schmitz, L. Sonnenschein, J. Steggemann, D. Teyssier 
RWTH Aachen University, III. Physikalisches Institut B, Aachen, Germany M. Bontenackels, M. Davids, M. Duda, G. Flügge, H. Geenen, M. Giffels, W. Haj Ahmad, D. Heydhausen, T. Kress, Y. Kuessel, A. Linn, A. Nowack, L. Perchalla, O. Pooth, J. Rennefeld, P. Sauerland, A. Stahl, M. Thomas, D. Tornier, M.H. Zoeller

\section{Deutsches Elektronen-Synchrotron, Hamburg, Germany}

M. Aldaya Martin, W. Behrenhoff, U. Behrens, M. Bergholz ${ }^{8}$, K. Borras, A. Campbell, E. Castro, D. Dammann, G. Eckerlin, A. Flossdorf, G. Flucke, A. Geiser, I. Glushkov, J. Hauk, H. Jung, M. Kasemann, I. Katkov, P. Katsas, C. Kleinwort, H. Kluge, A. Knutsson, D. Krücker, E. Kuznetsova, W. Lange, W. Lohmann ${ }^{8}$, R. Mankel, M. Marienfeld, I.-A. Melzer-Pellmann, A.B. Meyer, J. Mnich, A. Mussgiller, J. Olzem, A. Parenti, A. Raspereza, A. Raval, R. Schmidt ${ }^{8}$, T. Schoerner-Sadenius, N. Sen, M. Stein, J. Tomaszewska, D. Volyanskyy, R. Walsh, C. Wissing

\section{University of Hamburg, Hamburg, Germany}

C. Autermann, S. Bobrovskyi, J. Draeger, D. Eckstein, H. Enderle, U. Gebbert, K. Kaschube, G. Kaussen, R. Klanner, B. Mura, S. Naumann-Emme, F. Nowak, N. Pietsch, C. Sander, H. Schettler, P. Schleper, M. Schröder, T. Schum, J. Schwandt, A.K. Srivastava, H. Stadie, G. Steinbrück, J. Thomsen, R. Wolf

\section{Institut für Experimentelle Kernphysik, Karlsruhe, Germany}

J. Bauer, V. Buege, A. Cakir, T. Chwalek, D. Daeuwel, W. De Boer, A. Dierlamm, G. Dirkes, M. Feindt, J. Gruschke, C. Hackstein, F. Hartmann, M. Heinrich, H. Held, K.H. Hoffmann, S. Honc, T. Kuhr, D. Martschei, S. Mueller, Th. Müller, M.B. Neuland, M. Niegel, O. Oberst, A. Oehler, J. Ott, T. Peiffer, D. Piparo, G. Quast, K. Rabbertz, F. Ratnikov, M. Renz, A. Sabellek, C. Saout, A. Scheurer, P. Schieferdecker, F.-P. Schilling, G. Schott, H.J. Simonis, F.M. Stober, D. Troendle, J. Wagner-Kuhr, M. Zeise, V. Zhukov ${ }^{9}$, E.B. Ziebarth

\section{Institute of Nuclear Physics "Demokritos", Aghia Paraskevi, Greece}

G. Daskalakis, T. Geralis, S. Kesisoglou, A. Kyriakis, D. Loukas, I. Manolakos, A. Markou, C. Markou, C. Mavrommatis, E. Petrakou

\section{University of Athens, Athens, Greece}

L. Gouskos, T. Mertzimekis, A. Panagiotou ${ }^{1}$

University of Ioánnina, Ioánnina, Greece

I. Evangelou, P. Kokkas, N. Manthos, I. Papadopoulos, V. Patras, F.A. Triantis

KFKI Research Institute for Particle and Nuclear Physics, Budapest, Hungary A. Aranyi, G. Bencze, L. Boldizsar, G. Debreczeni, C. Hajdu ${ }^{1}$, D. Horvath ${ }^{10}$, A. Kapusi, K. Krajczar ${ }^{11}$, A. Laszlo, F. Sikler, G. Vesztergombi ${ }^{11}$

\section{Institute of Nuclear Research ATOMKI, Debrecen, Hungary}

N. Beni, J. Molnar, J. Palinkas, Z. Szillasi, V. Veszpremi

University of Debrecen, Debrecen, Hungary

P. Raics, Z.L. Trocsanyi, B. Ujvari 
Panjab University, Chandigarh, India

S. Bansal, S.B. Beri, V. Bhatnagar, M. Jindal, M. Kaur, J.M. Kohli, M.Z. Mehta, N. Nishu, L.K. Saini, A. Sharma, R. Sharma, A.P. Singh, J.B. Singh, S.P. Singh

University of Delhi, Delhi, India

S. Ahuja, S. Bhattacharya, S. Chauhan, B.C. Choudhary, P. Gupta, S. Jain, S. Jain, A. Kumar, R.K. Shivpuri

Bhabha Atomic Research Centre, Mumbai, India

R.K. Choudhury, D. Dutta, S. Kailas, S.K. Kataria, A.K. Mohanty ${ }^{1}$, L.M. Pant, P. Shukla, P. Suggisetti

Tata Institute of Fundamental Research - EHEP, Mumbai, India

T. Aziz, M. Guchait ${ }^{12}$, A. Gurtu, M. Maity ${ }^{13}$, D. Majumder, G. Majumder, K. Mazumdar, G.B. Mohanty, A. Saha, K. Sudhakar, N. Wickramage

Tata Institute of Fundamental Research - HECR, Mumbai, India

S. Banerjee, S. Dugad, N.K. Mondal

Institute for Studies in Theoretical Physics \& Mathematics (IPM), Tehran, Iran

H. Arfaei, H. Bakhshiansohi, S.M. Etesami, A. Fahim, M. Hashemi, A. Jafari, M. Khakzad, A. Mohammadi, M. Mohammadi Najafabadi, S. Paktinat Mehdiabadi, B. Safarzadeh, M. Zeinali

INFN Sezione di Bari ${ }^{a}$, Università di Bari ${ }^{b}$, Politecnico di Bari ${ }^{c}$, Bari, Italy

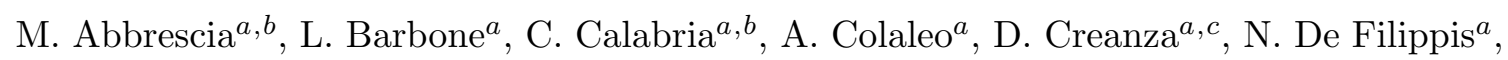
M. De Palma ${ }^{a, b}$, A. Dimitrov ${ }^{a}$, F. Fedele ${ }^{a}$, L. Fiore ${ }^{a}$, G. Iaselli ${ }^{a, c}$, L. Lusito ${ }^{a, b, 1}$,

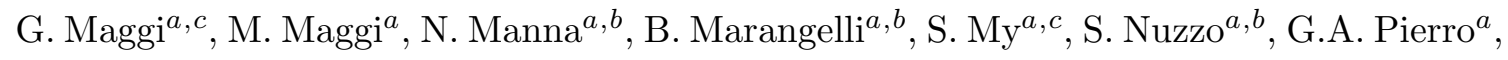
A. Pompili ${ }^{a, b}$, G. Pugliese ${ }^{a, c}$, F. Romano ${ }^{a, c}$, G. Roselli ${ }^{a, b}$, G. Selvaggi ${ }^{a, b}$, L. Silvestris ${ }^{a}$, R. Trentadue ${ }^{a}$, S. Tupputi ${ }^{a, b}$, G. Zito ${ }^{a}$

INFN Sezione di Bologna ${ }^{a}$, Università di Bologna ${ }^{b}$, Bologna, Italy

G. Abbiendi ${ }^{a}$, A.C. Benvenuti ${ }^{a}$, D. Bonacorsi ${ }^{a}$, S. Braibant-Giacomelli $^{a}, b$, P. Capiluppi $^{a, b}{ }$,

A. Castro ${ }^{a, b}$, F.R. Cavallo ${ }^{a}$, M. Cuffiani ${ }^{a, b}$, G.M. Dallavalle ${ }^{a}$, F. Fabbri ${ }^{a}$, A. Fanfani ${ }^{a, b}$,

D. Fasanella ${ }^{a}$, P. Giacomelli ${ }^{a}$, M. Giunta ${ }^{a}$, C. Grandi ${ }^{a}$, S. Marcellini ${ }^{a}$, M. Meneghelli ${ }^{a}, b$,

A. Montanari ${ }^{a}$, F.L. Navarria ${ }^{a, b}$, F. Odorici ${ }^{a}$, A. Perrotta ${ }^{a}$, A.M. Rossi ${ }^{a, b}$, T. Rovelli ${ }^{a, b}$,

G. Siroli ${ }^{a, b}$, R. Travaglini ${ }^{a, b}$

INFN Sezione di Catania ${ }^{a}$, Università di Catania ${ }^{b}$, Catania, Italy

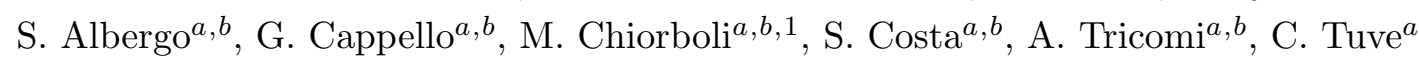

INFN Sezione di Firenze ${ }^{a}$, Università di Firenze ${ }^{b}$, Firenze, Italy

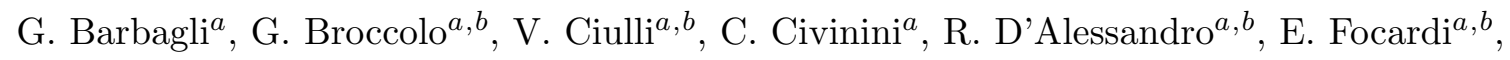

S. Frosali ${ }^{a, b}$, E. Gallo ${ }^{a}$, P. Lenzi ${ }^{a, b}$, M. Meschini ${ }^{a}$, S. Paoletti ${ }^{a}$, G. Sguazzoni ${ }^{a}$,

A. Tropiano ${ }^{a, 1}$

INFN Laboratori Nazionali di Frascati, Frascati, Italy

L. Benussi, S. Bianco, S. Colafranceschi ${ }^{14}$, F. Fabbri, D. Piccolo 
INFN Sezione di Genova, Genova, Italy

P. Fabbricatore, R. Musenich

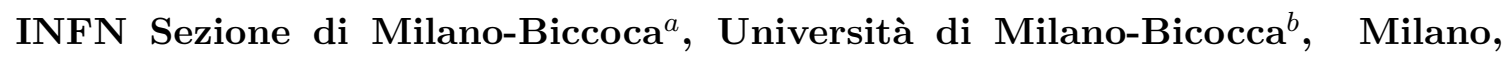
Italy

A. Benaglia ${ }^{a, b}$, G.B. Cerati ${ }^{a, b}$, F. De Guio ${ }^{a, b, 1}$, L. Di Matteo ${ }^{a, b}$, A. Ghezzi ${ }^{a, b, 1}$, P. Govoni ${ }^{a, b}$, M. Malberti ${ }^{a, b}$, S. Malvezzi ${ }^{a}$, A. Martelli ${ }^{a, b}$, A. Massironi ${ }^{a}, b$, D. Menasce ${ }^{a}$,

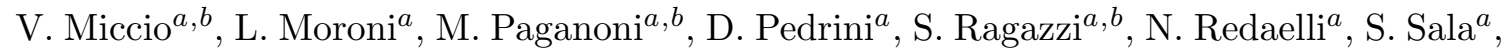
T. Tabarelli de Fatis ${ }^{a, b}$, V. Tancini ${ }^{a, b}$

INFN Sezione di Napoli ${ }^{a}$, Università di Napoli "Federico II" $b$, Napoli, Italy

S. Buontempo ${ }^{a}$, C.A. Carrillo Montoya $^{a}$, A. Cimmino ${ }^{a, b}$, A. De Cosa ${ }^{a, b, 1}$, M. De

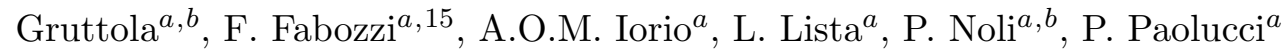

INFN Sezione di Padova ${ }^{a}$, Università di Padova ${ }^{b}$, Università di Trento (Trento) ${ }^{c}$, Padova, Italy

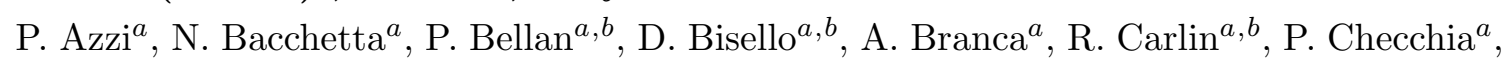

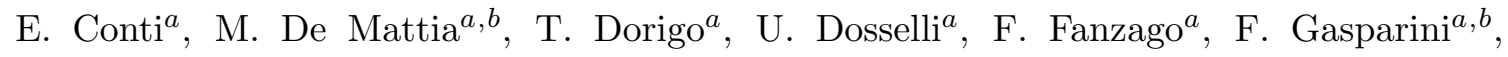
U. Gasparini ${ }^{a, b}$, P. Giubilato ${ }^{a, b}$, A. Gresele ${ }^{a, c}$, S. Lacaprara ${ }^{a, 16}$, I. Lazzizzera ${ }^{a, c}$, M. Margoni ${ }^{a, b}$, M. Mazzucato ${ }^{a}$, A.T. Meneguzzo ${ }^{a, b}$, L. Perrozzi ${ }^{a, 1}$, N. Pozzobon ${ }^{a, b}$, P. Ronchese ${ }^{a, b}$, F. Simonetto ${ }^{a, b}$, E. Torassa ${ }^{a}$, M. Tosi ${ }^{a, b}$, S. Vanini ${ }^{a, b}$, P. Zotto $^{a, b}$, G. Zumerle ${ }^{a, b}$

INFN Sezione di Pavia ${ }^{a}$, Università di Pavia ${ }^{b}$, Pavia, Italy

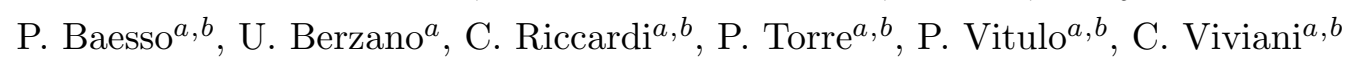

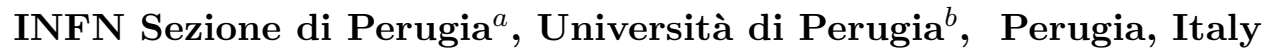

M. Biasini ${ }^{a, b}$, G.M. Bilei ${ }^{a}$, B. Caponeri ${ }^{a, b}$, L. Fanò ${ }^{a, b}$, P. Lariccia ${ }^{a, b}$, A. Lucaroni ${ }^{a, b}, 1$, G. Mantovani ${ }^{a}, b$, M. Menichelli ${ }^{a}$, A. Nappi ${ }^{a, b}$, A. Santocchia ${ }^{a, b}$, L. Servoli ${ }^{a}$, S. Taroni ${ }^{a, b}$, M. Valdata ${ }^{a, b}$, R. Volpe ${ }^{a, b, 1}$

INFN Sezione di Pisa ${ }^{a}$, Università di $\mathrm{Pisa}^{b}$, Scuola Normale Superiore di Pisa ${ }^{c}$, Pisa, Italy

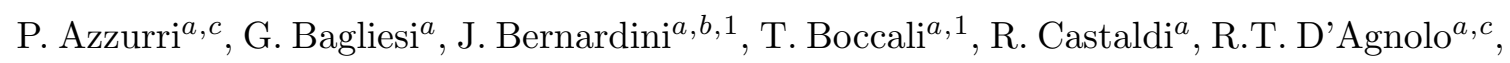

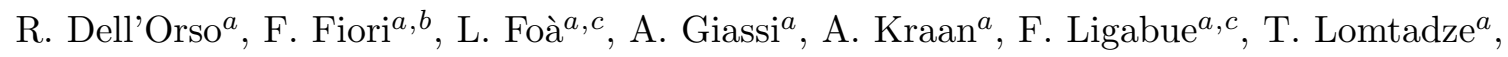
L. Martini ${ }^{a}$, A. Messineo ${ }^{a, b}$, F. Palla ${ }^{a}$, F. Palmonari ${ }^{a}$, S. Sarkar ${ }^{a, c}$, G. Segneri ${ }^{a}$, A.T. Serban ${ }^{a}$, P. Spagnolo ${ }^{a}$, R. Tenchini ${ }^{a, 1}$, G. Tonelli ${ }^{a, b, 1}$, A. Venturi ${ }^{a}$, P.G. Verdini ${ }^{a}$

INFN Sezione di Roma ${ }^{a}$, Università di Roma "La Sapienza" L. Barone ${ }^{a, b}$, F. Cavallari ${ }^{a, 1}$, D. Del Re ${ }^{a, b}$, E. Di Marco ${ }^{a, b}$, M. Diemoz ${ }^{a}$, D. Franci ${ }^{a, b}$, M. Grassi ${ }^{a}$, E. Longo ${ }^{a, b}$, G. Organtini ${ }^{a, b}$, A. Palma ${ }^{a, b}$, F. Pandolfi ${ }^{a, b}, 1$, R. Paramatti ${ }^{a}$, S. Rahatlou ${ }^{a, b, 1}$

INFN Sezione di Torino ${ }^{a}$, Università di Torino ${ }^{b}$, Università del Piemonte Orientale (Novara) ${ }^{c}$, Torino, Italy

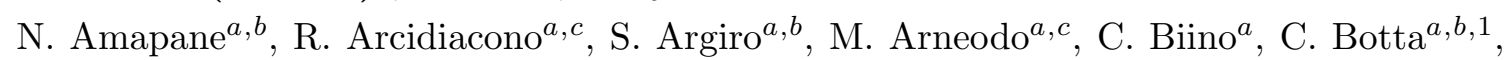

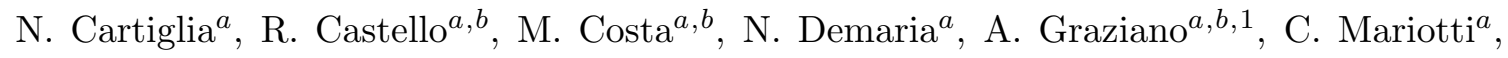


M. Marone ${ }^{a, b}$, S. Maselli ${ }^{a}$, E. Migliore ${ }^{a, b}$, G. Mila ${ }^{a, b}$, V. Monaco ${ }^{a, b}$, M. Musich ${ }^{a, b}$, M.M. Obertino ${ }^{a, c}$, N. Pastrone ${ }^{a}$, M. Pelliccioni ${ }^{a, b, 1}$, A. Romero ${ }^{a, b}$, M. Ruspa ${ }^{a, c}$, R. Sacchi ${ }^{a, b}$, V. Sola ${ }^{a, b}$, A. Solano ${ }^{a, b}$, A. Staiano ${ }^{a}$, D. Trocino ${ }^{a, b}$, A. Vilela Pereira ${ }^{a, b, 1}$

INFN Sezione di Trieste ${ }^{a}$, Università di Trieste ${ }^{b}$, Trieste, Italy

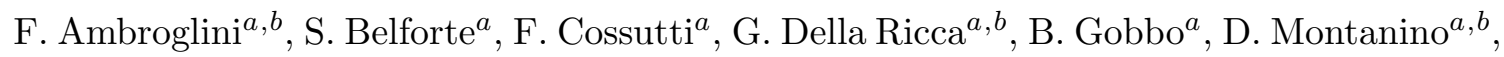
A. Penzo ${ }^{a}$

Kangwon National University, Chunchon, Korea

S.G. Heo

Kyungpook National University, Daegu, Korea

S. Chang, J. Chung, D.H. Kim, G.N. Kim, J.E. Kim, D.J. Kong, H. Park, D. Son, D.C. Son Chonnam National University, Institute for Universe and Elementary Particles, Kwangju, Korea

Zero Kim, J.Y. Kim, S. Song

Korea University, Seoul, Korea

S. Choi, B. Hong, M. Jo, H. Kim, J.H. Kim, T.J. Kim, K.S. Lee, D.H. Moon, S.K. Park, H.B. Rhee, E. Seo, S. Shin, K.S. Sim

University of Seoul, Seoul, Korea

M. Choi, S. Kang, H. Kim, C. Park, I.C. Park, S. Park, G. Ryu

Sungkyunkwan University, Suwon, Korea

Y. Choi, Y.K. Choi, J. Goh, J. Lee, S. Lee, H. Seo, I. Yu

Vilnius University, Vilnius, Lithuania

M.J. Bilinskas, I. Grigelionis, M. Janulis, D. Martisiute, P. Petrov, T. Sabonis

Centro de Investigacion y de Estudios Avanzados del IPN, Mexico City, Mexico

H. Castilla Valdez, E. De La Cruz Burelo, R. Lopez-Fernandez, A. Sánchez Hernández, L.M. Villasenor-Cendejas

Universidad Iberoamericana, Mexico City, Mexico

S. Carrillo Moreno, F. Vazquez Valencia

Benemerita Universidad Autonoma de Puebla, Puebla, Mexico

H.A. Salazar Ibarguen

Universidad Autónoma de San Luis Potosí, San Luis Potosí, Mexico

E. Casimiro Linares, A. Morelos Pineda, M.A. Reyes-Santos

University of Auckland, Auckland, New Zealand

P. Allfrey, D. Krofcheck, J. Tam

University of Canterbury, Christchurch, New Zealand

P.H. Butler, R. Doesburg, H. Silverwood

National Centre for Physics, Quaid-I-Azam University, Islamabad, Pakistan

M. Ahmad, I. Ahmed, M.I. Asghar, H.R. Hoorani, W.A. Khan, T. Khurshid, S. Qazi 
Institute of Experimental Physics, Warsaw, Poland

M. Cwiok, W. Dominik, K. Doroba, A. Kalinowski, M. Konecki, J. Krolikowski

\section{Soltan Institute for Nuclear Studies, Warsaw, Poland}

T. Frueboes, R. Gokieli, M. Górski, M. Kazana, K. Nawrocki, M. Szleper, G. Wrochna, P. Zalewski

Laboratório de Instrumentação e Física Experimental de Partículas, Lisboa, Portugal

N. Almeida, A. David, P. Faccioli, P.G. Ferreira Parracho, M. Gallinaro, P. Martins, G. Mini, P. Musella, A. Nayak, L. Raposo, P.Q. Ribeiro, J. Seixas, P. Silva, D. Soares, J. Varela ${ }^{1}$, H.K. Wöhri

Joint Institute for Nuclear Research, Dubna, Russia

I. Belotelov, P. Bunin, M. Finger, M. Finger Jr., I. Golutvin, A. Kamenev, V. Karjavin, G. Kozlov, A. Lanev, P. Moisenz, V. Palichik, V. Perelygin, S. Shmatov, V. Smirnov, A. Volodko, A. Zarubin

Petersburg Nuclear Physics Institute, Gatchina (St Petersburg), Russia

N. Bondar, V. Golovtsov, Y. Ivanov, V. Kim, P. Levchenko, V. Murzin, V. Oreshkin, I. Smirnov, V. Sulimov, L. Uvarov, S. Vavilov, A. Vorobyev

Institute for Nuclear Research, Moscow, Russia

Yu. Andreev, S. Gninenko, N. Golubev, M. Kirsanov, N. Krasnikov, V. Matveev, A. Pashenkov, A. Toropin, S. Troitsky

Institute for Theoretical and Experimental Physics, Moscow, Russia

V. Epshteyn, V. Gavrilov, V. Kaftanov ${ }^{\dagger}$, M. Kossov ${ }^{1}$, A. Krokhotin, S. Kuleshov, N. Lychkovskaya, A. Oulianov, G. Safronov, S. Semenov, I. Shreyber, V. Stolin, E. Vlasov, A. Zhokin

\section{Moscow State University, Moscow, Russia}

E. Boos, M. Dubinin ${ }^{17}$, L. Dudko, A. Ershov, A. Gribushin, O. Kodolova, I. Lokhtin, S. Obraztsov, S. Petrushanko, L. Sarycheva, V. Savrin, A. Snigirev

P.N. Lebedev Physical Institute, Moscow, Russia

V. Andreev, M. Azarkin, I. Dremin, M. Kirakosyan, S.V. Rusakov, A. Vinogradov

State Research Center of Russian Federation, Institute for High Energy Physics, Protvino, Russia

I. Azhgirey, S. Bitioukov, V. Grishin ${ }^{1}$, V. Kachanov, D. Konstantinov, V. Krychkine, V. Petrov, R. Ryutin, S. Slabospitsky, A. Sobol, L. Tourtchanovitch, S. Troshin, N. Tyurin, A. Uzunian, A. Volkov

University of Belgrade, Faculty of Physics and Vinca Institute of Nuclear Sciences, Belgrade, Serbia

P. Adzic ${ }^{18}$, M. Djordjevic, D. Krpic ${ }^{18}$, D. Maletic, J. Milosevic, J. Puzovic ${ }^{18}$ 
Centro de Investigaciones Energéticas Medioambientales y Tecnológicas (CIEMAT), Madrid, Spain

M. Aguilar-Benitez, J. Alcaraz Maestre, P. Arce, C. Battilana, E. Calvo, M. Cepeda, M. Cerrada, N. Colino, B. De La Cruz, C. Diez Pardos, C. Fernandez Bedoya, J.P. Fernández Ramos, A. Ferrando, J. Flix, M.C. Fouz, P. Garcia-Abia, O. Gonzalez Lopez, S. Goy Lopez, J.M. Hernandez, M.I. Josa, G. Merino, J. Puerta Pelayo, I. Redondo, L. Romero, J. Santaolalla, C. Willmott

Universidad Autónoma de Madrid, Madrid, Spain

C. Albajar, G. Codispoti, J.F. de Trocóniz

Universidad de Oviedo, Oviedo, Spain

J. Cuevas, J. Fernandez Menendez, S. Folgueras, I. Gonzalez Caballero, L. Lloret Iglesias, J.M. Vizan Garcia

Instituto de Física de Cantabria (IFCA), CSIC-Universidad de Cantabria, Santander, Spain

I.J. Cabrillo, A. Calderon, M. Chamizo Llatas, S.H. Chuang, I. Diaz Merino, C. Diez Gonzalez, J. Duarte Campderros, M. Felcini ${ }^{19}$, M. Fernandez, G. Gomez, J. Gonzalez Sanchez, R. Gonzalez Suarez, C. Jorda, P. Lobelle Pardo, A. Lopez Virto, J. Marco, R. Marco, C. Martinez Rivero, F. Matorras, J. Piedra Gomez ${ }^{20}$, T. Rodrigo, A. Ruiz Jimeno, L. Scodellaro, M. Sobron Sanudo, I. Vila, R. Vilar Cortabitarte

CERN, European Organization for Nuclear Research, Geneva, Switzerland

D. Abbaneo, E. Auffray, P. Baillon, A.H. Ball, D. Barney, F. Beaudette ${ }^{3}$, A.J. Bell ${ }^{21}$, D. Benedetti, C. Bernet ${ }^{3}$, A.K. Bhattacharyya, W. Bialas, P. Bloch, A. Bocci, S. Bolognesi, H. Breuker, G. Brona, K. Bunkowski, T. Camporesi, E. Cano, A. Cattai, G. Cerminara, T. Christiansen, J.A. Coarasa Perez, R. Covarelli, B. Curé, D. D’Enterria, T. Dahms, A. De Roeck, A. Elliott-Peisert, W. Funk, A. Gaddi, S. Gennai, G. Georgiou, H. Gerwig, D. Gigi, K. Gill, D. Giordano, F. Glege, R. Gomez-Reino Garrido, M. Gouzevitch, S. Gowdy, L. Guiducci, M. Hansen, J. Harvey, J. Hegeman, B. Hegner, C. Henderson, H.F. Hoffmann, A. Honma, V. Innocente, P. Janot, E. Karavakis, P. Lecoq, C. Leonidopoulos, C. Lourenço, A. Macpherson, T. Mäki, L. Malgeri, M. Mannelli, L. Masetti, F. Meijers, S. Mersi, E. Meschi, R. Moser, M.U. Mozer, M. Mulders, E. Nesvold ${ }^{1}$, L. Orsini, E. Perez, A. Petrilli, A. Pfeiffer, M. Pierini, M. Pimiä, G. Polese, A. Racz, G. Rolandi ${ }^{22}$, C. Rovelli ${ }^{23}$, M. Rovere, H. Sakulin, C. Schäfer, C. Schwick, I. Segoni, A. Sharma, P. Siegrist, M. Simon, P. Sphicas ${ }^{24}$, D. Spiga, M. Spiropulu ${ }^{17}$, F. Stöckli, M. Stoye, P. Tropea, A. Tsirou, G.I. Veres ${ }^{11}$, P. Vichoudis, M. Voutilainen, W.D. Zeuner

\section{Paul Scherrer Institut, Villigen, Switzerland}

W. Bertl, K. Deiters, W. Erdmann, K. Gabathuler, R. Horisberger, Q. Ingram, H.C. Kaestli, S. König, D. Kotlinski, U. Langenegger, F. Meier, D. Renker, T. Rohe, J. Sibille ${ }^{25}$, A. Starodumov ${ }^{26}$ 
Institute for Particle Physics, ETH Zurich, Zurich, Switzerland

L. Caminada ${ }^{27}$, Z. Chen, S. Cittolin, G. Dissertori, M. Dittmar, J. Eugster, K. Freudenreich, C. Grab, A. Hervé, W. Hintz, P. Lecomte, W. Lustermann, C. Marchica ${ }^{27}$, P. Martinez Ruiz del Arbol ${ }^{28}$, P. Meridiani, P. Milenovic ${ }^{29}$, F. Moortgat, A. Nardulli, P. Nef, F. NessiTedaldi, L. Pape, F. Pauss, T. Punz, A. Rizzi, F.J. Ronga, L. Sala, A.K. Sanchez, M.C. Sawley, B. Stieger, L. Tauscher ${ }^{\dagger}$, A. Thea, K. Theofilatos, D. Treille, C. Urscheler, R. Wallny ${ }^{19}$, M. Weber, L. Wehrli, J. Weng

Universität Zürich, Zurich, Switzerland

E. Aguiló, C. Amsler, V. Chiochia, S. De Visscher, C. Favaro, M. Ivova Rikova, A. Jaeger, B. Millan Mejias, C. Regenfus, P. Robmann, T. Rommerskirchen, A. Schmidt, H. Snoek, L. Wilke

\section{National Central University, Chung-Li, Taiwan}

Y.H. Chang, K.H. Chen, W.T. Chen, S. Dutta, A. Go, C.M. Kuo, S.W. Li, W. Lin, M.H. Liu, Z.K. Liu, Y.J. Lu, J.H. Wu, S.S. Yu

National Taiwan University (NTU), Taipei, Taiwan

P. Bartalini, P. Chang, Y.H. Chang, Y.W. Chang, Y. Chao, K.F. Chen, W.-S. Hou, Y. Hsiung, K.Y. Kao, Y.J. Lei, R.-S. Lu, J.G. Shiu, Y.M. Tzeng, M. Wang, J.T. Wei

\section{Cukurova University, Adana, Turkey}

A. Adiguzel, M.N. Bakirci, S. Cerci ${ }^{30}$, Z. Demir, C. Dozen, I. Dumanoglu, E. Eskut, S. Girgis, G. Gökbulut, Y. Güler, E. Gurpinar, I. Hos, E.E. Kangal, T. Karaman, A. Kayis Topaksu, A. Nart, G. Önengüt, K. Ozdemir, S. Ozturk, A. Polatöz, K. Sogut ${ }^{31}$, B. Tali, H. Topakli, D. Uzun, L.N. Vergili, M. Vergili, C. Zorbilmez

Middle East Technical University, Physics Department, Ankara, Turkey

I.V. Akin, T. Aliev, S. Bilmis, M. Deniz, H. Gamsizkan, A.M. Guler, K. Ocalan, A. Ozpineci, M. Serin, R. Sever, U.E. Surat, E. Yildirim, M. Zeyrek

Bogazici University, Istanbul, Turkey

M. Deliomeroglu, D. Demir ${ }^{32}$, E. Gülmez, A. Halu, B. Isildak, M. Kaya ${ }^{33}$, O. Kaya ${ }^{33}$, M. Özbek, S. Ozkorucuklu ${ }^{34}$, N. Sonmez ${ }^{35}$

National Scientific Center, Kharkov Institute of Physics and Technology, Kharkov, Ukraine

L. Levchuk

\section{University of Bristol, Bristol, United Kingdom}

P. Bell, F. Bostock, J.J. Brooke, T.L. Cheng, D. Cussans, R. Frazier, J. Goldstein, M. Grimes, M. Hansen, G.P. Heath, H.F. Heath, C. Hill, B. Huckvale, J. Jackson, L. Kreczko, S. Metson, D.M. Newbold ${ }^{36}$, K. Nirunpong, A. Poll, V.J. Smith, S. Ward

\section{Rutherford Appleton Laboratory, Didcot, United Kingdom}

L. Basso, K.W. Bell, A. Belyaev, C. Brew, R.M. Brown, B. Camanzi, D.J.A. Cockerill, J.A. Coughlan, K. Harder, S. Harper, B.W. Kennedy, E. Olaiya, D. Petyt, B.C. RadburnSmith, C.H. Shepherd-Themistocleous, I.R. Tomalin, W.J. Womersley, S.D. Worm 


\section{Imperial College, London, United Kingdom}

R. Bainbridge, G. Ball, J. Ballin, R. Beuselinck, O. Buchmuller, D. Colling, N. Cripps, M. Cutajar, G. Davies, M. Della Negra, C. Foudas, J. Fulcher, D. Futyan, A. Guneratne Bryer, G. Hall, Z. Hatherell, J. Hays, G. Iles, G. Karapostoli, L. Lyons, A.-M. Magnan, J. Marrouche, R. Nandi, J. Nash, A. Nikitenko ${ }^{26}$, A. Papageorgiou, M. Pesaresi, K. Petridis, M. Pioppi ${ }^{37}$, D.M. Raymond, N. Rompotis, A. Rose, M.J. Ryan, C. Seez, P. Sharp, A. Sparrow, A. Tapper, S. Tourneur, M. Vazquez Acosta, T. Virdee ${ }^{1}$, S. Wakefield, D. Wardrope, T. Whyntie

\section{Brunel University, Uxbridge, United Kingdom}

M. Barrett, M. Chadwick, J.E. Cole, P.R. Hobson, A. Khan, P. Kyberd, D. Leslie, W. Martin, I.D. Reid, L. Teodorescu

Baylor University, Waco, USA

K. Hatakeyama

\section{Boston University, Boston, USA}

T. Bose, E. Carrera Jarrin, A. Clough, C. Fantasia, A. Heister, J. St. John, P. Lawson, D. Lazic, J. Rohlf, L. Sulak

\section{Brown University, Providence, USA}

J. Andrea, A. Avetisyan, S. Bhattacharya, J.P. Chou, D. Cutts, S. Esen, A. Ferapontov, U. Heintz, S. Jabeen, G. Kukartsev, G. Landsberg, M. Narain, D. Nguyen, M. Segala, T. Speer, K.V. Tsang

\section{University of California, Davis, Davis, USA}

M.A. Borgia, R. Breedon, M. Calderon De La Barca Sanchez, D. Cebra, M. Chertok, J. Conway, P.T. Cox, J. Dolen, R. Erbacher, E. Friis, W. Ko, A. Kopecky, R. Lander, H. Liu, S. Maruyama, T. Miceli, M. Nikolic, D. Pellett, J. Robles, T. Schwarz, M. Searle, J. Smith, M. Squires, M. Tripathi, R. Vasquez Sierra, C. Veelken

\section{University of California, Los Angeles, Los Angeles, USA}

V. Andreev, K. Arisaka, D. Cline, R. Cousins, A. Deisher, J. Duris, S. Erhan, C. Farrell, J. Hauser, M. Ignatenko, C. Jarvis, C. Plager, G. Rakness, P. Schlein ${ }^{\dagger}$, J. Tucker, V. Valuev

\section{University of California, Riverside, Riverside, USA}

J. Babb, R. Clare, J. Ellison, J.W. Gary, F. Giordano, G. Hanson, G.Y. Jeng, S.C. Kao, F. Liu, H. Liu, A. Luthra, H. Nguyen, G. Pasztor ${ }^{38}$, A. Satpathy, B.C. Shen ${ }^{\dagger}$, R. Stringer, J. Sturdy, S. Sumowidagdo, R. Wilken, S. Wimpenny

\section{University of California, San Diego, La Jolla, USA}

W. Andrews, J.G. Branson, E. Dusinberre, D. Evans, F. Golf, A. Holzner, R. Kelley, M. Lebourgeois, J. Letts, B. Mangano, J. Muelmenstaedt, S. Padhi, C. Palmer, G. Petrucciani, H. Pi, M. Pieri, R. Ranieri, M. Sani, V. Sharma ${ }^{1}$, S. Simon, Y. Tu, A. Vartak, F. Würthwein, A. Yagil 


\section{University of California, Santa Barbara, Santa Barbara, USA}

D. Barge, R. Bellan, C. Campagnari, M. D'Alfonso, T. Danielson, P. Geffert, J. Incandela, C. Justus, P. Kalavase, S.A. Koay, D. Kovalskyi, V. Krutelyov, S. Lowette, N. Mccoll, V. Pavlunin, F. Rebassoo, J. Ribnik, J. Richman, R. Rossin, D. Stuart, W. To, J.R. Vlimant, M. Witherell

\section{California Institute of Technology, Pasadena, USA}

A. Bornheim, J. Bunn, Y. Chen, M. Gataullin, D. Kcira, V. Litvine, Y. Ma, A. Mott, H.B. Newman, C. Rogan, K. Shin, V. Timciuc, P. Traczyk, J. Veverka, R. Wilkinson, Y. Yang, R.Y. Zhu

\section{Carnegie Mellon University, Pittsburgh, USA}

B. Akgun, A. Calamba, R. Carroll, T. Ferguson, Y. Iiyama, D.W. Jang, S.Y. Jun, Y.F. Liu, M. Paulini, J. Russ, N. Terentyev, H. Vogel, I. Vorobiev

\section{University of Colorado at Boulder, Boulder, USA}

J.P. Cumalat, M.E. Dinardo, B.R. Drell, C.J. Edelmaier, W.T. Ford, B. Heyburn, E. Luiggi Lopez, U. Nauenberg, J.G. Smith, K. Stenson, K.A. Ulmer, S.R. Wagner, S.L. Zang

\section{Cornell University, Ithaca, USA}

L. Agostino, J. Alexander, F. Blekman, A. Chatterjee, S. Das, N. Eggert, L.J. Fields, L.K. Gibbons, B. Heltsley, K. Henriksson, W. Hopkins, A. Khukhunaishvili, B. Kreis, V. Kuznetsov, Y. Liu, G. Nicolas Kaufman, J.R. Patterson, D. Puigh, D. Riley, A. Ryd, M. Saelim, X. Shi, W. Sun, W.D. Teo, J. Thom, J. Thompson, J. Vaughan, Y. Weng, P. Wittich

\section{Fairfield University, Fairfield, USA}

A. Biselli, G. Cirino, D. Winn

\section{Fermi National Accelerator Laboratory, Batavia, USA}

S. Abdullin, M. Albrow, J. Anderson, G. Apollinari, M. Atac, J.A. Bakken, S. Banerjee, L.A.T. Bauerdick, A. Beretvas, J. Berryhill, P.C. Bhat, I. Bloch, F. Borcherding, K. Burkett, J.N. Butler, V. Chetluru, H.W.K. Cheung, F. Chlebana, S. Cihangir, M. Demarteau, D.P. Eartly, V.D. Elvira, I. Fisk, J. Freeman, Y. Gao, E. Gottschalk, D. Green, K. Gunthoti, O. Gutsche, A. Hahn, J. Hanlon, R.M. Harris, J. Hirschauer, E. James, H. Jensen, M. Johnson, U. Joshi, R. Khatiwada, B. Kilminster, B. Klima, K. Kousouris, S. Kunori, S. Kwan, P. Limon, R. Lipton, J. Lykken, K. Maeshima, J.M. Marraffino, D. Mason, P. McBride, T. McCauley, T. Miao, K. Mishra, S. Mrenna, Y. Musienko ${ }^{39}$, C. NewmanHolmes, V. O’Dell, S. Popescu, R. Pordes, O. Prokofyev, N. Saoulidou, E. Sexton-Kennedy, S. Sharma, A. Soha, W.J. Spalding, L. Spiegel, P. Tan, L. Taylor, S. Tkaczyk, L. Uplegger, E.W. Vaandering, R. Vidal, J. Whitmore, W. Wu, F. Yang, F. Yumiceva, J.C. Yun

\section{University of Florida, Gainesville, USA}

D. Acosta, P. Avery, D. Bourilkov, M. Chen, G.P. Di Giovanni, D. Dobur, A. Drozdetskiy, R.D. Field, M. Fisher, Y. Fu, I.K. Furic, J. Gartner, S. Goldberg, B. Kim, S. Klimenko, J. Konigsberg, A. Korytov, K. Kotov, A. Kropivnitskaya, T. Kypreos, K. Matchev, 
G. Mitselmakher, L. Muniz, Y. Pakhotin, M. Petterson, C. Prescott, R. Remington, M. Schmitt, B. Scurlock, P. Sellers, M. Snowball, D. Wang, J. Yelton, M. Zakaria

Florida International University, Miami, USA

C. Ceron, V. Gaultney, L. Kramer, L.M. Lebolo, S. Linn, P. Markowitz, G. Martinez, D. Mesa, J.L. Rodriguez

Florida State University, Tallahassee, USA

T. Adams, A. Askew, J. Bochenek, J. Chen, B. Diamond, S.V. Gleyzer, J. Haas, S. Hagopian, V. Hagopian, M. Jenkins, K.F. Johnson, H. Prosper, S. Sekmen, V. Veeraraghavan

\section{Florida Institute of Technology, Melbourne, USA}

M.M. Baarmand, B. Dorney, S. Guragain, M. Hohlmann, H. Kalakhety, R. Ralich, I. Vodopiyanov

University of Illinois at Chicago (UIC), Chicago, USA

M.R. Adams, I.M. Anghel, L. Apanasevich, Y. Bai, V.E. Bazterra, R.R. Betts, J. Callner, R. Cavanaugh, C. Dragoiu, E.J. Garcia-Solis, C.E. Gerber, D.J. Hofman, S. Khalatyan, F. Lacroix, C. O'Brien, E. Shabalina, C. Silvestre, A. Smoron, D. Strom, N. Varelas

The University of Iowa, Iowa City, USA

U. Akgun, E.A. Albayrak, B. Bilki, K. Cankocak ${ }^{40}$, W. Clarida, F. Duru, C.K. Lae, E. McCliment, J.-P. Merlo, H. Mermerkaya, A. Mestvirishvili, A. Moeller, J. Nachtman, C.R. Newsom, E. Norbeck, J. Olson, Y. Onel, F. Ozok, S. Sen, J. Wetzel, T. Yetkin, K. Yi Johns Hopkins University, Baltimore, USA

B.A. Barnett, B. Blumenfeld, A. Bonato, C. Eskew, D. Fehling, G. Giurgiu, A.V. Gritsan, Z.J. Guo, G. Hu, P. Maksimovic, S. Rappoccio, M. Swartz, N.V. Tran, A. Whitbeck

The University of Kansas, Lawrence, USA

P. Baringer, A. Bean, G. Benelli, O. Grachov, M. Murray, D. Noonan, V. Radicci, S. Sanders, J.S. Wood, V. Zhukova

Kansas State University, Manhattan, USA

D. Bandurin, T. Bolton, I. Chakaberia, A. Ivanov, M. Makouski, Y. Maravin, S. Shrestha, I. Svintradze, Z. Wan

Lawrence Livermore National Laboratory, Livermore, USA

J. Gronberg, D. Lange, D. Wright

University of Maryland, College Park, USA

A. Baden, M. Boutemeur, S.C. Eno, D. Ferencek, J.A. Gomez, N.J. Hadley, R.G. Kellogg, M. Kirn, Y. Lu, A.C. Mignerey, K. Rossato, P. Rumerio, F. Santanastasio, A. Skuja, J. Temple, M.B. Tonjes, S.C. Tonwar, E. Twedt

\section{Massachusetts Institute of Technology, Cambridge, USA}

B. Alver, G. Bauer, J. Bendavid, W. Busza, E. Butz, I.A. Cali, M. Chan, V. Dutta, P. Everaerts, G. Gomez Ceballos, M. Goncharov, K.A. Hahn, P. Harris, Y. Kim, M. Klute, 
Y.-J. Lee, W. Li, C. Loizides, P.D. Luckey, T. Ma, S. Nahn, C. Paus, C. Roland, G. Roland, M. Rudolph, G.S.F. Stephans, K. Sumorok, K. Sung, E.A. Wenger, B. Wyslouch, S. Xie, M. Yang, Y. Yilmaz, A.S. Yoon, M. Zanetti

University of Minnesota, Minneapolis, USA

P. Cole, S.I. Cooper, P. Cushman, B. Dahmes, A. De Benedetti, P.R. Dudero, G. Franzoni, J. Haupt, K. Klapoetke, Y. Kubota, J. Mans, V. Rekovic, R. Rusack, M. Sasseville, A. Singovsky

University of Mississippi, University, USA

L.M. Cremaldi, R. Godang, R. Kroeger, L. Perera, R. Rahmat, D.A. Sanders, D. Summers University of Nebraska-Lincoln, Lincoln, USA

K. Bloom, S. Bose, J. Butt, D.R. Claes, A. Dominguez, M. Eads, J. Keller, T. Kelly, I. Kravchenko, J. Lazo-Flores, C. Lundstedt, H. Malbouisson, S. Malik, G.R. Snow

State University of New York at Buffalo, Buffalo, USA

U. Baur, A. Godshalk, I. Iashvili, A. Kharchilava, A. Kumar, K. Smith, J. Zennamo

Northeastern University, Boston, USA

G. Alverson, E. Barberis, D. Baumgartel, O. Boeriu, M. Chasco, K. Kaadze, S. Reucroft, J. Swain, D. Wood, J. Zhang

Northwestern University, Evanston, USA

A. Anastassov, A. Kubik, N. Odell, R.A. Ofierzynski, B. Pollack, A. Pozdnyakov, M. Schmitt, S. Stoynev, M. Velasco, S. Won

University of Notre Dame, Notre Dame, USA

L. Antonelli, D. Berry, M. Hildreth, C. Jessop, D.J. Karmgard, J. Kolb, T. Kolberg, K. Lannon, W. Luo, S. Lynch, N. Marinelli, D.M. Morse, T. Pearson, R. Ruchti, J. Slaunwhite, N. Valls, J. Warchol, M. Wayne, J. Ziegler

The Ohio State University, Columbus, USA

B. Bylsma, L.S. Durkin, J. Gu, P. Killewald, T.Y. Ling, M. Rodenburg, G. Williams

Princeton University, Princeton, USA

N. Adam, E. Berry, P. Elmer, D. Gerbaudo, V. Halyo, P. Hebda, A. Hunt, J. Jones,

E. Laird, D. Lopes Pegna, D. Marlow, T. Medvedeva, M. Mooney, J. Olsen, P. Piroué,

H. Saka, D. Stickland, C. Tully, J.S. Werner, A. Zuranski

University of Puerto Rico, Mayaguez, USA

J.G. Acosta, X.T. Huang, A. Lopez, H. Mendez, S. Oliveros, J.E. Ramirez Vargas, A. Zatserklyaniy

\section{Purdue University, West Lafayette, USA}

E. Alagoz, V.E. Barnes, G. Bolla, L. Borrello, D. Bortoletto, A. Everett, A.F. Garfinkel,

Z. Gecse, L. Gutay, M. Jones, O. Koybasi, A.T. Laasanen, N. Leonardo, C. Liu, V. Maroussov, M. Meier, P. Merkel, D.H. Miller, N. Neumeister, K. Potamianos, I. Shipsey, D. Silvers, A. Svyatkovskiy, H.D. Yoo, J. Zablocki, Y. Zheng 


\section{Purdue University Calumet, Hammond, USA}

P. Jindal, N. Parashar

\section{Rice University, Houston, USA}

C. Boulahouache, V. Cuplov, K.M. Ecklund, F.J.M. Geurts, J.H. Liu, J. Morales, B.P. Padley, R. Redjimi, J. Roberts, J. Zabel

\section{University of Rochester, Rochester, USA}

B. Betchart, A. Bodek, Y.S. Chung, P. de Barbaro, R. Demina, Y. Eshaq, H. Flacher,

A. Garcia-Bellido, P. Goldenzweig, Y. Gotra, J. Han, A. Harel, D.C. Miner, D. Orbaker, G. Petrillo, D. Vishnevskiy, M. Zielinski

\section{The Rockefeller University, New York, USA}

A. Bhatti, L. Demortier, K. Goulianos, G. Lungu, C. Mesropian, M. Yan

\section{Rutgers, the State University of New Jersey, Piscataway, USA}

O. Atramentov, A. Barker, D. Duggan, Y. Gershtein, R. Gray, E. Halkiadakis, D. Hidas,

D. Hits, A. Lath, S. Panwalkar, R. Patel, A. Richards, K. Rose, S. Schnetzer, S. Somalwar,

R. Stone, S. Thomas

\section{University of Tennessee, Knoxville, USA}

G. Cerizza, M. Hollingsworth, S. Spanier, Z.C. Yang, A. York

\section{Texas A\&M University, College Station, USA}

J. Asaadi, R. Eusebi, J. Gilmore, A. Gurrola, T. Kamon, V. Khotilovich, R. Montalvo, C.N. Nguyen, J. Pivarski, A. Safonov, S. Sengupta, A. Tatarinov, D. Toback, M. Weinberger

Texas Tech University, Lubbock, USA

N. Akchurin, C. Bardak, J. Damgov, C. Jeong, K. Kovitanggoon, S.W. Lee, P. Mane, Y. Roh, A. Sill, I. Volobouev, R. Wigmans, E. Yazgan

\section{Vanderbilt University, Nashville, USA}

E. Appelt, E. Brownson, D. Engh, C. Florez, W. Gabella, W. Johns, P. Kurt, C. Maguire, A. Melo, P. Sheldon, J. Velkovska

\section{University of Virginia, Charlottesville, USA}

M.W. Arenton, M. Balazs, S. Boutle, M. Buehler, S. Conetti, B. Cox, B. Francis, R. Hirosky, A. Ledovskoy, C. Lin, C. Neu, T. Patel, R. Yohay

\section{Wayne State University, Detroit, USA}

S. Gollapinni, R. Harr, P.E. Karchin, V. Loggins, M. Mattson, C. Milstène, A. Sakharov

\section{University of Wisconsin, Madison, USA}

M. Anderson, M. Bachtis, J.N. Bellinger, D. Carlsmith, S. Dasu, J. Efron, L. Gray, K.S. Grogg, M. Grothe, R. Hall-Wilton ${ }^{1}$, M. Herndon, P. Klabbers, J. Klukas, A. Lanaro, C. Lazaridis, J. Leonard, J. Liu, D. Lomidze, R. Loveless, A. Mohapatra, W. Parker, D. Reeder, I. Ross, A. Savin, W.H. Smith, J. Swanson, M. Weinberg 
$\dagger$ : Deceased

1: Also at CERN, European Organization for Nuclear Research, Geneva, Switzerland

2: Also at Universidade Federal do ABC, Santo Andre, Brazil

3: Also at Laboratoire Leprince-Ringuet, Ecole Polytechnique, IN2P3-CNRS, Palaiseau, France

4: Also at Suez Canal University, Suez, Egypt

5: Also at Fayoum University, El-Fayoum, Egypt

6: Also at Soltan Institute for Nuclear Studies, Warsaw, Poland

7: Also at Université de Haute-Alsace, Mulhouse, France

8: Also at Brandenburg University of Technology, Cottbus, Germany

9: Also at Moscow State University, Moscow, Russia

10: Also at Institute of Nuclear Research ATOMKI, Debrecen, Hungary

11: Also at Eötvös Loránd University, Budapest, Hungary

12: Also at Tata Institute of Fundamental Research - HECR, Mumbai, India

13: Also at University of Visva-Bharati, Santiniketan, India

14: Also at Facoltà Ingegneria Università di Roma "La Sapienza", Roma, Italy

15: Also at Università della Basilicata, Potenza, Italy

16: Also at Laboratori Nazionali di Legnaro dell' INFN, Legnaro, Italy

17: Also at California Institute of Technology, Pasadena, USA

18: Also at Faculty of Physics of University of Belgrade, Belgrade, Serbia

19: Also at University of California, Los Angeles, Los Angeles, USA

20: Also at University of Florida, Gainesville, USA

21: Also at Université de Genève, Geneva, Switzerland

22: Also at Scuola Normale e Sezione dell' INFN, Pisa, Italy

23: Also at INFN Sezione di Roma; Università di Roma "La Sapienza", Roma, Italy

24: Also at University of Athens, Athens, Greece

25: Also at The University of Kansas, Lawrence, USA

26: Also at Institute for Theoretical and Experimental Physics, Moscow, Russia

27: Also at Paul Scherrer Institut, Villigen, Switzerland

28: Also at Instituto de Física de Cantabria (IFCA), CSIC-Universidad de Cantabria, Santander, Spain

29: Also at University of Belgrade, Faculty of Physics and Vinca Institute of Nuclear Sciences, Belgrade, Serbia

30: Also at Adiyaman University, Adiyaman, Turkey

31: Also at Mersin University, Mersin, Turkey

32: Also at Izmir Institute of Technology, Izmir, Turkey

33: Also at Kafkas University, Kars, Turkey

34: Also at Suleyman Demirel University, Isparta, Turkey

35: Also at Ege University, Izmir, Turkey

36: Also at Rutherford Appleton Laboratory, Didcot, United Kingdom

37: Also at INFN Sezione di Perugia; Università di Perugia, Perugia, Italy

38: Also at KFKI Research Institute for Particle and Nuclear Physics, Budapest, Hungary

39: Also at Institute for Nuclear Research, Moscow, Russia

40: Also at Istanbul Technical University, Istanbul, Turkey 